\title{
Enjeux sociaux et politiques de la « vulgarisation scientifique » en Révolution (1780-1810)
}

Jean-Luc Chappey

\section{(2) OpenEdition \\ 1 Journals}

Édition électronique

URL : https://journals.openedition.org/ahrf/1578

DOI : $10.4000 /$ ahrf. 1578

ISSN : 1952-403X

\section{Éditeur :}

Armand Colin, Société des études robespierristes

\section{Édition imprimée}

Date de publication : 1 décembre 2004

Pagination : 11-51

ISSN : 0003-4436

\section{Référence électronique}

Jean-Luc Chappey, «Enjeux sociaux et politiques de la « vulgarisation scientifique » en Révolution (1780-1810) », Annales historiques de la Révolution française [En ligne], 338 | octobre-décembre 2004 mis en ligne le 20 février 2006, consulté le 22 avril 2022. URL : http://journals.openedition.org/ahrf/ 1578 ; DOI : https://doi.org/10.4000/ahrf.1578

Ce document a été généré automatiquement le 22 avril 2022.

Tous droits réservés 


\title{
Enjeux sociaux et politiques de la "vulgarisation scientifique » en Révolution (1780-1810)
}

\author{
Jean-Luc Chappey
}

1 La «vulgarisation ", considérée comme un système de processus sociaux et discursifs, fait l'objet, depuis plusieurs années, d'une attention nouvelle de la part des historiens. Paradoxalement, et alors que des recherches novatrices ouvrent de nouvelles voies pour les XVIIIe et XIXe siècle, la période révolutionnaire reste, à quelques exceptions près, à l'écart de ce mouvement d'ensemble. Sans doute cette constatation historiographique peut-elle s'expliquer en grande partie par l'ambiguïté de la notion de «vulgarisation ». Dans le domaine intellectuel ou artistique, la période révolutionnaire est traversée de phénomènes que l'on rassemble traditionnellement sous la notion d'acculturation ${ }^{~}$. Sciences, lettres et arts constituent les éléments, parmi d'autres, d'une entreprise générale de formation du citoyen et de régénération sociale et politique. Dans cette optique, les approches concernant la diffusion des savoirs scientifiques et techniques ont privilégié les études de certaines institutions ou

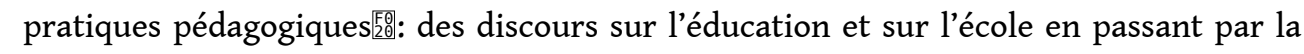
presse ou les grandes institutions (Muséum d'histoire naturelle, École de l'an III, École Polytechnique ou École nationale des Arts et Métiers), l'historiographie s'est surtout concentrée, d'un côté, sur les supports de la diffusion des savoirs et, de l'autre, sur les pratiques (expériences, etc.) et les discours qui en sont les instruments. L'approche en terme de «vulgarisation» a été délaissée ou traitée de façon isolée, parfois anecdotique: pendant une période où la "vulgarisation " des savoirs semble être partout, elle est, au final, nulle part. Certes, l'utilisation même de la notion n'est pas sans poser quelques problèmes, particulièrement lorsque l'on cherche à vouloir la comprendre à la lumière des logiques générales qui traversent l'espace scientifique. Le risque, dans lequel tombent souvent les études, est de vouloir considérer la «vulgarisation » et ses acteurs sous l'angle d'un système de pratiques et de productions particulier et autonome : à travers une réification de phénomènes particulièrement bien connus pour le XIXe siècle (au cours duquel la « vulgarisation » s'institutionnalise 
à travers des discours, des supports et des lieux spécifiques ${ }^{2}$ ), la "vulgarisation " apparaît, de manière parfois caricaturale, sous une forme qui n'est ni contextualisée ni problématisée. Cet article ne prétend donc pas présenter un catalogue exhaustif des différents acteurs, supports et discours hétéroclites que l'on pourrait rattacher à la "vulgarisation". Son objet est plutôt de s'interroger sur les enjeux scientifiques, politiques et sociaux de l'écriture de la science qui s'inscrit toujours par rapport à un public, plus ou moins large. À partir de l'analyse des luttes et des débats qui mettent au prise des conceptions opposées du public entre les années 1780 et 1810 , je voudrais justement discuter de la signification et de la validité heuristique de la notion de "vulgarisation ». En me plaçant dans une histoire sociale et politique des sciences, je montrerai que la question de la diffusion des sciences sous la Révolution permet, non seulement de repenser les débats fondamentaux qui traversent le monde des sciences, mais aussi de préciser les relations entre le monde des sciences et l'ordre politique et social. C'est donc en quelque sorte "en creux» que j'étudierais la progressive institutionnalisation d'une " vulgarisation » entre la fin de l'Ancien Régime et l'Empire, une institutionnalisation liée à un régime particulier de la science française qui va se renforcer pendant tout le XIXe siècle. C'est en effet à partir de l'Empire, au moment où l'on assiste justement au renforcement du processus de professionnalisation des sciences, que l'on peut constater l'émergence de la figure sociale du "vulgarisateur » qui acquiert un statut autonome et se dote des outils particuliers (journaux, etc.) de son intervention dans l'espace public: la "vulgarisation» scientifique s'impose alors comme une pratique (sociale et discursive) de «traduction » et de " médiation » entre le champ scientifique et le grand public.

2 Il est admis que le XVIIIe siècle constitue un moment particulièrement favorable à la diffusion et au "partage des savoirs " à travers des pratiques dont les modalités ne sauraient être distinguées de celles de la production scientifique et de la "science normale ». Selon Hélène Meztger, "un caractère tout à fait remarquable de cette période heureuse pour la pensée que fut le XVIIIe siècle, c'est que la science qui s'enseigne, la science qui se vulgarise, la science qui se met 'à la portée de tout le monde' est à la hauteur de la science qui se fait " ${ }^{3}$. En dépit des débats qu'a pu susciter une telle affirmation ${ }^{4}$, il semble que l'élargissement du champ des savoirs et le progrès des connaissances aient été suivis d'un élargissement du public scientifique, d'une dilatation de l'horizon d'attente à laquelle les scientifiques auraient répondu. L'abandon des langues anciennes au profit des langues vulgaires dans les publications scientifiques, l'essor du livre de science dans le volume global des productions imprimées apparaissent comme autant de signes d'un mouvement général qui, en France comme dans la plupart des États européens, marquerait le succès de la vulgarisation scientifique et la mise en place d'un marché éditorial spécifique, comme en témoigne la vogue entourant les ouvrages qui accompagnent la multiplication des cabinets de curiosité et de physique, mais aussi les nombreux traités publiés sous le titre d'»Amusements et jeux mathématiques et physiques » ou de "Récréations physiques ". L'essor des périodiques et la fortune commerciale des ouvrages destinés à un " large " public ${ }^{5}$ illustrent encore ce processus qui ne se dément pas tout au long du siècle et dans lequel s'inscrivent les stratégies éditoriales menées successivement par Le Breton et Panckoucke autour des éditions de l'Encyclopédie. Désormais, «le livre scientifique n'est plus destiné au seul monde des savants. De très nombreux ouvrages de vulgarisation apparaissent publiés pour un vaste public de non-spécialistes " ${ }^{6}$. Des aérostats aux expériences sur l'électricité en passant par la curiosité qui entoure la 
botanique, le "triomphe de la science » et l'enthousiasme suscité par ses spectacles ${ }^{7}$ caractérisaient ainsi l'émergence d'un public d' "amateurs " 8 dont les savants, parmi les plus éminents, viendraient combler l'attente sous une forme "aimable " et " plaisante ». Il convient néanmoins de s'interroger sur la signification et les enjeux de ce processus que l'on présente sous la notion de « vulgarisation » et qui tend à imposer l'idée selon laquelle le progrès des sciences se serait naturellement accompagné au XVIIIe siècle d'un processus d'ouverture vers un large public, mouvement qui s'inscrirait dans "l'idéal pédagogique » des Lumières. Les travaux de Jeffrey S. Ravel sur les parterres des théâtres ${ }^{9}$ et de Thomas Crow sur les Salons organisés par l'Académie de peinture au XVIIIe siècle ${ }^{10}$ nous amènent à nous interroger plus précisément sur les conflits importants qui rythment l'émergence du "public » et les transformations des modalités de productions et de jugement intellectuel dans l'espace scientifique. Si l'horizon d'attente n'existe jamais en dehors des productions qui en fixent les principes et si le "public » n'émerge comme instance de jugement qu'à travers des porte-parole qui en dessinent les frontières selon des intérêts particuliers, la question de la «vulgarisation » doit être reposée à la lumière des formes sociales de la production des savoirs et des conditions épistémologiques et méthodologiques d'un régime de scientificité spécifique. "Ecrire pour un large public », faire de la science de manière "plaisante » et " aimable » dans des «théâtres de société » ne sont pas des pratiques auxiliaires auxquelles se livreraient des savants soucieux d'élargir leur audience : la "vulgarisation » et la «science normale " participent en effet de la même logique et s'inscrivent dans le même espace ${ }^{11}$. Comme le montrent les analyses portant sur les querelles qui traversent le monde des arts, mais aussi l'espace politique ${ }^{12}$, le " public » apparaît comme un outil polémique dans des discours qui s'opposent et, parlà, tendent à en redéfinir chaque fois les contours. La construction d'un public spécifique (par le jeu des préfaces par exemple) renvoie à des prises de position qui s'inscrivent dans des stratégies, individuelles ou collectives, en vue de la conquête d'une légitimité scientifique et institutionnelle et le contrôle des instruments, des objets et des discours d'autorité ${ }^{13}$. De ce fait, la question posée par le "public" s'intègre dans les rapports de force et de domination qui traversent la communauté scientifique d'une période, rapports que l'usage souvent intempestif du terme de "vulgarisation » tend trop souvent à masquer. Au lieu de constater un mouvement allant dans le sens d'un apparent "élargissement» du public scientifique et de se contenter de cataloguer les lieux et les supports d'une vulgarisation qui serait toujours plus importante, il convient plutôt de s'interroger sur les règles sociales et cognitives de l'espace scientifique du XVIIIe siècle et ainsi de mettre en lumière les tensions et les conflits qui émergent, particulièrement autour des années 1780, autour des usages de la catégorie de « public » dans les débats qui traversent l'espace savant.

I. Le conflit entre « science mondaine » et " science sévère "

3 Jusque dans les années 1780 , le régime de scientificité sur lequel sont fondées les règles de légitimité des productions savantes et les normes de la validité de la "vérité» scientifique peut être défini sous la notion de «science mondaine». Par-là, nous entendons aussi bien un système de principes épistémologiques et méthodologiques qu'une configuration sociale au sein de laquelle s'inscrivent les normes de production, de validation et de diffusion des savoirs. Les travaux de Steven Shapin et de Simon Schaffer pour l'Angleterre du XVIIe siècle ${ }^{14}$ et de Christian Licoppe pour la France du XVIIe et du XVIIIe siècle ${ }^{15}$, ont décrit le fonctionnement de cette "science mondaine " qui prend assise sur un public choisi et restreint (les gentlemen ou les «hommes 
éclairés ", archétypes des "hommes désintéressés »), des lieux spécifiques (les "salons" ou les public rooms) et des normes de légitimation particulière (la preuve "sensible ») qui se déploie dans une "rhétorique scientifique». Dans ce système théorique et social de production du savoir scientifique, le "public », composé d'une élite «mixte» d'hommes "éclairés» et de savants (les fameux « amateurs»), joue un rôle important, non comme mécène ou simple spectateur, mais comme instance essentielle de validation des connaissances et de légitimation du savant. Ce que l'on entend ici par "vulgarisation" n'est autre chose que la recherche nécessaire d'une adéquation entre les productions, les pratiques scientifiques et un "public» qui confère la reconnaissance savante et qui renforce, de manière dialectique, son statut de public "éclairé » par le biais des discours tenus par les savants qui se produisent devant lui et qui tendent à valoriser ses compétences de jugement. Une connaissance est en effet jugée authentique et avérée lorsqu'elle accède à cet espace public, mais un espace public particulier : c'est la condition de personne éclairée et la relation de confiance qui la lie avec le savant qui fondent la validité des témoignages et « l'objectivité » de la connaissance expérimentale. S'adresser au public, et si possible au plus "large» public entendu toujours dans une conception restreinte et choisie, constitue donc un passage obligé pour construire une carrière scientifique au XVIIIe siècle. Dans ce processus, on comprend comment se construit la relation complexe entre les discours "scientifique » et "littéraire ». Souvent étudiée à la lumière d'une distinction qui s'impose progressivement à partir du début du XIXe siècle, l'écriture littéraire de la science est considérée comme le signe évident du travail de vulgarisation, partant du présupposé que le discours scientifique serait irréductiblement incompréhensible, ésotérique et non-littéraire. Or, là encore, il convient d'être particulièrement prudent. Au XVIIIe siècle, l'écriture de la science, même la plus "spécialisée ", impose le recours à des formes rhétoriques de discours que nous avons pris l'habitude de classer aujourd'hui comme littéraires. Le «beau style », la recherche d'une langue "plaisante » (qui apparaissent comme la «marque de fabrique » des hommes de science du XVIIIe siècle) ne reposent pas sur une recherche de pur agrément et ne peuvent en aucun cas être considérés comme les ornements d'un discours scientifique " sérieux ». Ce dispositif a été particulièrement bien mis en valeur par Marie-Françoise Mortureux dans son analyse des stratégies discursives employées par Fontenelle (1657-1757) dans ses Entretiens sur la pluralité des mondes (1686) ${ }^{16}$. MarieFrançoise Mortureux étudie les modalités de la construction d'un "double langage » destiné à convaincre aussi bien les "gens du monde », les " amateurs ", que les savants, «double langage» renvoyant aux exigences d'une «double reconnaissance» de la "vérité » scientifique et de la légitimité du savant. Cette configuration impose donc une manière particulière de faire et d'écrire la science que l'on retrouve, Christian Licoppe l'a particulièrement bien montré, dans les récits des expériences et dans la construction de la preuve scientifique: la preuve «spectaculaire» et "sensible» reposant sur une rhétorique littéraire qui met en scène et théâtralise l'intervention de l'énonciateur (" je vis avec la plus grande satisfaction », " étincelante », " ruisseaux de feux »...) et le regard du " public» illustre parfaitement le fonctionnement de cette science mondaine qui valorise le caractère «aimable » de la science dont l'abbé Nollet et ses cours au Collège de Navarre constituent, à partir des années 1760 , le meilleur exemple ${ }^{17}$. Or, à partir des années 1779 , on peut constater que le modèle social et cognitif de cette science mondaine est progressivement concurrencé par un autre modèle, celui que nous définissons sous la notion de « science sévère ». 
$4 \mathrm{Au}$ cours des années 1770, la «science mondaine » et ses plus illustres représentants subissent des attaques sur deux fronts, social et épistémologique. La contestation sociale peut être illustrée à travers l'exemple de Gilbert Romme (1750-1795) dont la correspondance permet d'éclairer sa trajectoire entre son arrivée à Paris en 1774 et son départ en Russie ${ }^{18}$. Jeune diplômé de mathématiques, Romme arrive dans la capitale avec l'ambition de devenir médecin. Muni de lettres de recommandation signées de ses amis auvergnats, il commence à frapper aux portes des personnalités savantes les plus influentes (d'Alembert, en particulier) afin d'obtenir le «droit d'entrée » au sein d'une sociabilité mondaine susceptible de lui offrir reconnaissance et protections. Or, rapidement, et en dépit des efforts financiers déployés pour se conformer à la « culture des apparences", Romme prend progressivement ses distances par rapport à une sociabilité mondaine dont Antoine Lilti a dévoilé récemment les ressorts ${ }^{19}$. Dans ses lettres, Romme critique ses représentants et, plus généralement, multiplie les attaques très acerbes contre les instruments de la preuve "spectaculaire ", révélant ainsi le regard peu complaisant qu'il porte sur ceux qui usent de la rhétorique "mondaine » (la science «polie ») et des ressources de la littérature dans le domaine de la science : «Le champ vaste de l'éloquence commence vous le savez où finit le district très borné de la raison. Quand on ne peut plus prouver, on veut émouvoir, on veut étonner les sens et l'oreille remplie des sons graves et harmonieux, on admire, on applaudit cet homme de génie ! C'est la lumière du siècle! Mais le sang froid calme un peu les sens, et l'homme de génie n'a pas le bon sens et l'on rougit d'avoir admiré ce qu'on ne comprenait pas. Son obscurité faisait tout son mérite ${ }^{20}$. Ne voyons pas là, dans la continuité d'Alessandro Galante Garrone les traits de la timidité d'un jeune provincial ou les restes d'une éducation janséniste ${ }^{21}$, mais plutôt une réaction contre les modalités d'une "science mondaine" dont de nombreux jeunes savants, comme lui, dénoncent le fonctionnement et le système de distinction qu'elle impose (c'est sans doute là qu'il convient de parler du «rousseauisme» de Romme). Cette réaction sociale contre la "science mondaine ", qui s'inscrit dans un mouvement plus large de contestation des "corps" et des communautés fonctionnant progressivement comme des espaces d'exclusion, participe à l'émergence dans les années 1770-1780 d'un nouveau régime de scientificité qui constitue ce que l'on appelle le «révolution scientifique » et ouvre la voie à la «science moderne ». C'est en effet autour, entre autres, de Louis-Bernard Guyton de Morveau (1737-1816) ${ }^{22}$, Antoine-Laurent Lavoisier (1734-1794) ${ }^{23}$ et René-Just Haüy (1745-1822) que se constitue un nouveau modèle d'intelligibilité opposé aux cosmogonies philosophiques, mais aussi un nouveau régime social de production des savoirs. Appuyé sur le recours à la mathématisation et aux classifications, l'émergence de la «science sévère" peut être considérée comme une réaction contre le fonctionnement d'une "science mondaine » qui instaure le jugement des amateurs "éclairés " en instance de légitimation et de validation du discours scientifique. Affirmant que les travaux en anatomie comparée ont été négligés du fait que ses objets seraient trop répugnants pour « plaire à ces amateurs qu'il faut captiver par l'élégance et la mobilité du spectacle » ${ }^{24}$, Félix Vicq d'Azyr (1748-1794) affirme que le progrès des connaissances n'est possible que si la science se détourne du public «mondain », et plus généralement des fondements de la production des savoirs fondés sur la mondanité. On assiste ainsi à une restriction de la notion de "public»: si les "amateurs" et " curieux» peuvent toujours assister aux expériences, ils sont désormais ravalés au statut de spectateurs de la science, à un rôle passif qui les empêchent d'intervenir dans le processus de construction du discours scientifique et de validation de la preuve dont 
les modalités apparaissent désormais comme les prérogatives exclusives des "spécialistes". On voit combien cette mise à distance du public mondain (celui des " amateurs» et des "dilettantes») est directement liée aux nouveaux principes d'intelligibilité (les classifications, les nomenclatures) et aux enjeux qui entourent la construction d'un nouveau langage scientifique qui tend progressivement à se revendiquer comme radicalement distinct de la littérature. Alors que les partisans de la "science mondaine » défendaient l'idée d'une science susceptible d'être comprise par les amateurs "éclairés ", les partisans de la science "sévère ", se référant à Condillac pour qui le progrès des sciences repose sur une réforme du langage, défendent la construction d'une langue scientifique au sein de laquelle le travail sur les terminologies compte autant que l'observation et l'analyse des phénomènes. De ce fait, ils imposent l'idée selon laquelle la validité et la légitimité de la "vérité "scientifique ne peuvent être reconnues et accordées que par des savants consacrés par les partisans de la « réforme » des sciences, réduisant ainsi l'espace de production de la science à une communauté de "pairs ». Comme le souligne Christian Licoppe à travers son analyse des récits d'expériences rédigés par Lavoisier et ses partisans ${ }^{25}$, cette rupture est essentielle pour comprendre les déplacements des espaces de sociabilité scientifique et l'affirmation des normes d'une preuve « exacte " (dévalorisation de la preuve visuelle, délocalisation de l'expérience, neutralité du locuteur...) aux dépens de la preuve «spectaculaire ». Les conflits autour de la nomenclature chimique de Lavoisier ou de l'introduction des méthodes de classification linnéenne en botanique ${ }^{26}$, la création en 1788 de la Société Philomathique et de la Société linnéenne, l'essor des journaux destinés aux seuls "spécialistes " (pensons aux Annales de chimie créées en 1783) sont autant de témoignages de la transformation qui s'opère dans le monde des sciences au cours des années 1780. Comme l'affirme Claude-Antoine-Gaspard Riche, fondateur de la Société Philomathique, les partisans de la « science sévère » se distinguent nettement des personnalités mondaines, «celles-ci se réunissent pour éclairer les autres, et nous pour nous instruire $"{ }^{27}$. L'enjeu touche au degré de légitimité, et plus encore, au statut même de savant que peuvent revendiquer les acteurs. La question de la définition d'un public scientifique qui se cristallise à partir des années 1780 dans le conflit entre "science mondaine " et "science sévère ", le débat porte non seulement sur la nature du public, mais aussi sur la distinction entre outsiders et insiders, entre " amateurs » et "professionnels " ${ }^{28}$. À bien des égards, cette période se caractérise par le retrait du scientifique hors de la «République des Lettres» et par l'émergence de l'idée d'une " communauté » savante, constituant par-là une étape importante dans le processus d'autonomisation de l'espace scientifique. Notons que cette transformation importante qui voit la promotion de la "science sévère " est en grande partie accélérée par la construction de nouveaux rapports entre l'État et le monde des sciences qui caractérisent les années 1770-1780. On assiste, pour des domaines de savoir particuliers comme la statistique et la médecine et dans des lieux spécifiques (l'Académie des sciences et la Société royale de médecine), à la valorisation de la science comme « institution d'État » et à la mise en place d'une nouvelle figure du savant considéré désormais comme expert. Comme l'a clairement montré Eric Brian ${ }^{29}$, la mise en place de cette nouvelle configuration, caractérisée par le rapprochement entre savants et administrateurs, repose sur la mobilisation des ressources d'une « science sévère » (les outils mathématiques, en particulier, qui s'imposent dans les enquêtes administratives et les topographies médicales) dont les représentants renforcent par ce biais leur position scientifique et institutionnelle ${ }^{30}$. 
5 Néanmoins, il convient d'insister sur ce point: dans les années 1780, rien n'est encore joué dans le conflit qui oppose la «science mondaine" à la "science sévère ». Les partisans de la « science sévère » sont d'autant plus virulents contre la mondanité qu'ils doivent, à travers leurs ouvrages, construire un nouveau public de spécialistes et imposer, par là, de nouvelles normes de savoir scientifique. La définition des contours du «public » scientifique est donc l'objet central des oppositions qui émergent aussi bien au sein de l'Académie des science qu'entre la Faculté de Médecine et la Société royale de médecine et l'enjeu des débats est bien le contrôle des règles d'autorité et de domination du savoir scientifique : le conflit porte en effet sur les destinataires du discours scientifique, sur les modes de diffusion, et, au final, sur la construction de la science elle-même. La contre-offensive menée par les partisans de la «science mondaine " est d'autant plus importante qu'elle renvoie à la question des profits financiers et symboliques. Pour ceux qui ne bénéficient pas des relations ou des protections suffisantes pour intégrer les nouveaux "laboratoires", s'adresser à un large public devient une condition essentielle pour tenter de se construire, ou de conserver, une réputation dans le monde des sciences ${ }^{31}$. Dans cette perspective, l'essor, dans les années 1780, des publications destinées aux «dames" prend valeur de symbole : le public féminin constitue, pour les partisans de la "science mondaine ", le signe de ce qui les distingue -sur le plan social et théorique- des tenants de la «science sévère ». Avec une nostalgie révélant le terrain perdu par les représentants de la "science mondaine", Joseph-Aignan Sigaud de La Fond rappelle ainsi qu'il doit sa renommée aux «dames »: "Quand je me rappelle ces heureuses circonstances de ma vie, je me plais à croire que ce fût à ce concours inhabituel des Dames, à ce désir ardent qu'elles témoignaient de s'instruire, à l'émulation qu'elles excitaient, que je fus redevable de cette multitude d'auditeurs distingués qui fréquentaient mon école et suivirent mes cours " ${ }^{32}$. On le constate, dans la lutte qui s'engage pour la maîtrise des outils de légitimité institutionnelle et théorique, ce que l'on a coutume de désigner sous le terme de "vulgarisation » va jouer un rôle crucial : comme dans la lutte autour des parterres et des Salons de peinture, le conflit au sein de l'espace scientifique se cristallise autour des différentes conceptions du "public». Si les représentants de la "science sévère" renforcent indéniablement leur position au sein des institutions scientifiques liés à l'appareil administratif, ils sont confrontés à d'importantes résistances, non seulement de la part des représentants de la "science mondaine", mais aussi de la part de ceux qui, favorables à l'exclusion du public «mondain » des modalités de production et de validation scientifique, critiquent la spécialisation et l'éclatement des savoirs que semble reconnaître la réforme de l'Académie royale des sciences en 1785. C'est le cas, par exemple, de l'abbé François Rozier qui, à travers le journal qu'il publie à partir de 1771 et qu'il destine aux "vrais savants ", Observations sur la physique, sur l'histoire naturelle et sur les arts, cherche à conserver un ordre " encyclopédique " des savoirs ${ }^{33}$. Comme Rozier, nombreux sont ceux qui défendent l'idée d'une science «utile » qui s'était imposée dès le début du XVIIIe siècle. Il s'agit pour eux de garantir la plus grande diffusion des résultats et des produits d'une science qu'ils refusent de cloisonner dans le seul espace de l'expérience. Plus précisément, ils combattent un processus qui voit s'accroître la distance entre, d'un côté, la production de la science et, de l'autre, sa validation par un public d'utilisateurs qu'ils recherchent du côté des ingénieurs ou des artisans. Considérant que la validation de la "vérité scientifique" repose sur l'expérimentation professionnelle, ils entendent ainsi maintenir le lien entre les savants et les gens de métiers. Or, avec la « science sévère », 
cette distance tend à s'imposer. L'écart entre la théorie et l'expérience qui, dans le régime de l'utilité ne pouvait être réduit, se trouve ici renforcé par la clôture complète de l'espace expérimental sur lui-même. Les portes du «laboratoire» se ferment ainsi aux " curieux ", mais aussi aux artisans. Le rôle nouveau attribué aux instruments de mesure et de calcul (et non plus à l'observation) comme supports de la preuve, le retrait de l'observateur qui ne doit plus interférer dans les résultats, marquent l'émergence d'une nouvelle rhétorique de la science où les chiffres (tableau, graphes) tiennent une place fondamentale: on se trouve dans un espace social renfermé autour d'expérimentateurs compétents et d'un système de la vérité scientifique autoréférentiel.

Dans ce combat contre la "science sévère", des alliances (parfois contre-nature) se créent autour des critiques contre la spécialisation des sciences et la construction d'une langue nouvelle qui visent à renforcer la position d'une science encyclopédique, utile et cosmogonique devant rester accessible au public «éclairé» (et non plus seulement "mondain»), conception que défend par exemple l'abbé Pierre Bertholon ${ }^{34}$. Cette tension qui caractérise les années pré-révolutionnaires me parait particulièrement importante dans l'épisode "mesmériste " ${ }^{35}$. Sans qu'il soit question ici de rouvrir un dossier qui mériterait pourtant de nouvelles recherches, il me semble en effet que les ambiguités et la complexité de ce mouvement peuvent être lus à travers le conflit qui oppose les tenants des différentes conceptions de la science mondaine/utile/sévère. On se saurait en effet comprendre la violence des débats et la grande complexité théorique et sociale qui caractérise ce mouvement sans le replacer dans le contexte particulier de l'exacerbation du conflit qui oppose les représentants de ces conceptions de la science. Il n'est en effet pas fortuit de constater que les ennemis de la «science sévère " (l'abbé Nollet, l'abbé Bertholon en particulier) voient dans le mesmérisme un cadre privilégié pour défendre les principes théoriques et sociaux de la science « mondaine » et « utile » quitte, pour les besoins de leur cause, à légitimer certaines " aberrations » entourant les expériences autour du fluide magnétique. Si cette position suscite l'hostilité la plus vive des membres de la Commission royale nommée en 1785 pour juger de la validité scientifique (Lavoisier, Bailly...), les critiques portent autant sur les fondements théoriques du mesmérisme que sur les modalités sociales de la production et de validation du savoir. Pour les partisans de la "science sévère », les mesméristes sont des « charlatans » moins parce qu'ils défendent des principes scientifiques qui seraient faux (dans le rapport qu'il rédige, Antoine-Laurent Jussieu, membre de la Commission royale, reconnaît la légitimité scientifique de certains présupposés mesmériens ; plus généralement, des partisans de la "science sévère », l'aliéniste Philippe Pinel par exemple, puiseront dans le mesmérisme des outils théoriques importants) que parce qu'ils s'opposent à l'exclusion du public des "amateurs » et des gens de métiers hors des frontières de la "science normale ». Selon les porte-parole de la "science sévère ", qui s'appuient sur la légitimité que leur confère l'État, la "vraie» science doit se cantonner à des espaces fermés, des « laboratoires » auxquels les " oisifs ", les artisans ou les dames ne peuvent plus avoir accès ${ }^{36}$. Or, et c'est ce qui rend ce mouvement mesmériste particulièrement complexe, le combat "science mondaine »/ "utile »vs " science sévère " se charge, dans ces années 1780 , d'enjeux politiques. Le mouvement est en effet investi également par les représentants de ce que Robert Darnton définit sous le titre de la «tendance radicale du mesmérisme », des acteurs (Marat, Brissot) en lutte autant contre la «science mondaine » que contre la "science sévère ». Pour eux, la science doit être "populaire » et ne saurait être réduite aux frontières du public 
restreint et choisi des "salons" ni au cercle des spécialistes consacrés par les institutions scientifiques. S'attaquant particulièrement aux académies et aux normes exclusives de la "science sévère ", ils défendent une nouvelle conception de la science qui instaure le "peuple», entendu ici comme la « Nation » en instance de jugement scientifique. Signe évident d'une politisation, l'émergence de la «Nation» définie comme destinataire privilégiée des productions et de découvertes, mais aussi, au nom de "l'utilité » comme juge de la "vérité » scientifique, légitime -jusqu'en 1793- les attaques contre les académies et transforme radicalement les enjeux du débat. Désormais, la "science sévère " doit se défendre contre une accusation d'autant plus violente qu'elle s'inscrit dans les attaques portées contre le "despotisme ministériel » et les autorités traditionnelles, celle d'une science spéculative (représentée en large partie par Lavoisier qui devient la cible privilégiée des attaques d'un Marat ${ }^{37}$ ) que les normes semblent placer au rang des ennemis de la Nation. Cette introduction d'une conception du public assimilé à la « Nation » dans le débat scientifique des années 1780 va jouer un rôle crucial pendant la Révolution française.

II. Les contradictions de la « science révolutionnaire "

7 À partir de 1789, l'affirmation des principes sur lesquels doit reposer le nouvel ordre politique a des conséquences importantes sur l'ordre scientifique. Erigé en fondement de la légitimité politique, le "peuple» se voit conférer une place éminente dans les discours savants. Les sciences et leurs représentants doivent désormais répondre aux exigences de la mission qui leur est conférée, celle d'» éclairer » le peuple et participer ainsi au renforcement et à la conservation de l'ordre politique et social. Or, si la très grande majorité des savants se conforme aux exigences de cette mission, sa mise en pratique pose problème et oppose encore, mais dans des termes neufs, les partisans de la «science mondaine » et " utile » à ceux de la "science sévère », conflit qui s'inscrit complètement dans la temporalité des luttes politiques. Dès 1790 , les conséquences des «libertés » nouvelles se font sentir dans le monde des sciences : de nombreux acteurs profitent du nouveau contexte pour investir l'espace des productions scientifiques et conquérir (ou renforcer) leur propre réputation ${ }^{38}$, phénomène qui aboutit à créer une très forte concurrence entre les acteurs et, de fait, à reposer la question de la légitimité du statut de savant, et plus généralement, du statut de la science. Ces débats dépassent largement le cadre des journaux et des ouvrages et tendent à occuper une position centrale dans les logiques cognitives et institutionnelles, individuelles et collectives, qui caractérisent l'espace scientifique. Le jeu des réseaux de solidarités qui se révèle à travers la création de nouvelles sociétés savantes (la Société nationale d'histoire naturelle en 1790) ou de journaux, tend progressivement à institutionnaliser les oppositions et à les radicaliser. Dès lors, les débats sur les modalités de production, de diffusion et de validation des savoirs - un temps neutralisé en 1789 - refont surface en 1790 en se cristallisant autour du problème de la «langue scientifique ». D'un côté, les partisans de la "raison classificatoire", des nomenclatures et du "style sévère " attaquent le " beau style » et critiquent ceux qui, à l'instar d'un Buffon, auraient succombé aux sirènes de la mondanité au risque de fragiliser la nature «exacte » de leur discours scientifique ${ }^{39}$. En 1790, Riche affirme ainsi que «le célèbre Buffon, plus poète que philosophe, dont le génie brillant, souvent séduit par les erreurs de son imagination, parvient si rarement à la vérité, trop simple pour le faste de ses expressions » 40[5:; rappelons encore les propos ironiques avec lesquels Condorcet rend hommage en 1790 à Buffon avec qui "l'histoire naturelle [est devenue] une connaissance presque vulgaire; elle fut pour toutes les classes de la société ou un 
amusement ou une occupation; on voulait avoir un cabinet comme on voulait avoir une bibliothèque $"{ }^{41}$. Selon eux, le progrès des sciences susceptible de raffermir l'entreprise politique, n'est possible qu'à partir de l'exclusion des « charlatans » et de la réunion des spécialistes, seuls détenteurs des instruments de contrôle de la production des savoirs. Dès lors, la construction d'une langue scientifique qui rompt avec la langue commune et « vulgaire » est selon eux une condition essentielle de ce progrès des connaissances. La création de la Société d'histoire naturelle ${ }^{42}$, la publication à partir de 1791 du Bulletin de la Société philomathique sont autant de témoignages d'une institutionnalisation de la «science sévère" présentée comme l'apanage des «vrais savants 》[1000: "Voilà pourquoi les vrais savants aiment les ouvrages concis; voilà pourquoi le style laconique et tout à la fois harmonieux et poétique de Linneus fait et fera toujours tant d'hommes solidement instruits. La société philomathique a senti l'importance de cette concision, lorsqu'elle a conçu le projet de bulletin destiné à mettre ses correspondants au courant de ses travaux et des découvertes faites autour d'elle " ${ }^{43}$. De l'autre côté, les partisans de la "science mondaine" défendent toujours l'écriture littéraire du discours scientifique et la participation du public "éclairé », et en particulier du public féminin, comme instance de jugement scientifique. Eux aussi se dotent des supports institutionnels nécessaires à mener leur combat. C'est ainsi que certains opposants à la chimie de Lavoisier investissent dès 1790 , la Société Nationale des Neuf Sœurs qui accordent une place importante au public des «dames " ${ }^{44}$. Citons le cas de JacquesFrançois Demachy (1728-1803) qui publie un « Examen impartial de la nouvelle doctrine des chimistes modernes ou pneumatiques» dans le Tribut de la Société, article dans lequel il s'en prend violemment à ceux qui créent des noms «barbares » et inventent une langue qu'il juge ésotérique en s'appuyant sur les mathématiques et sur l'utilisation, dans les expériences, de nouveaux instruments :

«Deux sortes de routes conduisent aux sciences: celle des faits, et celle de la théorie : deux sortes d'hommes, les artistes et les philosophes, suivent séparément chacune d'elles: la seconde est plus susceptible d'égarer celui qui s'y livre. Ainsi, tandis que le jardinier perfectionne son art, dirige ses serres avec plus d'économie et de succès, et parvient à multiplier les variétés par des insertions inattendues; tandis qu'il accélère les productions agréables, les jouissances utiles, et les multiplie, une foule de prétendants à la science botanique, puisent les subtilités de leur imagination, l'un, pour bâtir un système, qu'à peine lui-même il conçoit, l'autre, pour donner à l'art minutieux des physionomies végétales, une sorte de considération; celui-ci, pour forger des mots barbares, dont il croit embellir les fruits du hasard, en surcharge des plantes déjà connues, celui-là bas et cupide adulateur, croit honorer du nom d'un Mécène ignorant, des plantes éphémères et fortuites. Tous prétendent avoir perfectionné la botanique : ils l'ont rendue obscure et presque inintelligible [...]; que dirions-nous de notre physique actuelle, autrefois si simplement cultivée à l'aide d'instruments commodes et si peu compliqués? Ne la voyons-nous pas subordonnée à des machines, à des rouages sans nombre, à des embellissements fastueux qui, sous prétexte d'une plus exacte précision, portent dans l'esprit d'un observateur de bonne foi plus de doutes sur le succès réel de l'expérience ? [...] Pour reculer davantage les progrès de la science, un jargon hérissé de grec, boursouflé de latin, digne du sphinx thébain, la précision intégrale, un calcul décimal, sont venus occuper nos chimistes, et détourner de la véritable science tous ceux que le bon sens y appelait.» ${ }^{\text {is }}$

8 Dans ce débat au sein duquel les enjeux théoriques ne sont pas absents, il convient, selon Demachy, de briser la distance entre la science et le public, un public qui ne saurait plus se réduire désormais aux lieux de sociabilité aristocratiques, mais qui est toujours considéré comme choisi et restreint. Rejetant les classifications et la 
mathématisation, il tente de conserver la place de la science dans la culture «lettrée ». Face à la disparition des "mécènes " et des "salons", ces partisans de la "science mondaine » tentent de se tourner vers les nouvelles élites sociales pour en faire les destinataires privilégiés de la science. C'est d'ailleurs ce même public que visent encore les animateurs du Lycée de Paris en 1792, justifiant l'instauration d'un «droit d'entrée » susceptible de filtrer les « inconnus » : «Le prix de la souscription est de cent francs pour les hommes, et de cinquante francs pour les dames. On recevra, avec la quittance de cette somme, un billet d'entrée. Les citoyens souscripteurs sont instamment priés de vouloir s'assujettir à apporter leur billet chaque fois qu'ils se présenteront, quelqu'importune que puisse paraître cette règle; elle est nécessaire, puisqu'elle est liée au seul moyen d'écarter les inconnus. La formalité pour l'admission des nouveaux souscripteurs est d'avoir un présentateur connu [...]. Il n'y aura point de demi-souscriptions, ni aucune espèce d'entrée gratuite " ${ }^{46}$. On voit comment cette défense de la "science mondaine » s'inscrit dans les logiques politiques (en défendant une certaine conception du " peuple ») et, plus précisément, participe à la "guerre des mots » dans la continuité des attaques menées par les contre-révolutionnaires contre les nouveaux néologismes révolutionnaires ${ }^{47}$. Cette offensive tend progressivement à amalgamer, pour la dénoncer, la " révolution » des mots et des sciences et la révolution politique et à rapprocher la défense de la Révolution de celle de la "science sévère ". Dans le contexte de la mise en place des nouvelles mesures métriques et des poids et mesures (considérée comme le témoignage du caractère "jacobin» de la "science sévère »), la défense de la « science mondaine » se retrouve en effet placée dans le camp des adversaires de la Révolution. De ce fait, on peut comprendre que l'année 1792 ouvre une période de marginalisation progressive de la configuration de la "science mondaine » et, à l'inverse de "victoire " de la «science sévère ». Les difficultés (en particulier financières) liées à la conservation des lieux de sociabilité «mondaine », les contraintes politiques qui pèsent désormais sur les justifications à réduire la définition $\mathrm{du}$ «public » dans une conception restreinte et choisie, justifient le mouvement de retrait de la science «mondaine ». De nombreuses études ont justifié la «victoire » de la "science sévère" en affirmant que ses présupposés méthodologiques et épistémologiques étaient plus en adéquation (pensons aux débats sur la langue) avec la pensée politique "républicaine». Ce serait avec la «nouvelle chimie» ${ }^{48}$ ou la botanique linnéenne qui a moins pour but de découvrir les "substances" que de s'attacher à décrire les relations entre les éléments et les phénomènes naturels. Il convient également de prendre en compte le repositionnement du discours tenu par les partisans de la "science sévère »: dès 1789 , ils se réapproprient en quelque sorte le discours tenu par les membres de la tendance radicale du mesmérisme et affirment la mission politique d'une science destinée au service de la "Nation ». S'ils excluent le "public » et le «peuple» des modalités de production et de validation du savoir, ils n'en défendent pas moins une science qui doit être entièrement soumise aux attentes de la «Nation » et, plus précisément de l'État. Cette position n'est pas sans poser quelques problèmes à partir de 1792, au moment où les impératifs liés à la guerre vont transformer encore les rapports entre l'État et la science.

9 Le renforcement institutionnel des lieux de savoir où la «science se fait » semble en effet entrer en contradiction avec la mission d' "éclairer» le peuple. Cette contradiction apparaît d'ailleurs au grand jour dès 1792 au sujet des usages du jardin botanique et de la ménagerie du Jardin du Roi, institution qui dès 1789 est appelée par les autorités à jouer un rôle essentiel dans la promotion des nouveaux principes 
politiques ${ }^{49}$. Contre ceux qui revendiquent un accès et un usage "sauvage » du jardin pour le public de visiteurs, s'opposent les naturalistes du Jardin qui défendent l'idée d'un jardin comme lieu de savoir, et donc souhaitent en restreindre, du moins en contrôler, l'accès et l'usage ${ }^{50}$. Alors que quelques mois plus tôt le buste de Linné avait été cassé, Bernardin de Saint-Pierre, intendant du Jardin, se plaint, dans une lettre adressée au ministre Rolland à la fin de 1792, des dégradations causées par le public: « des agitateurs persuadent le peuple que le Jardin étant à la Nation, toute la Nation a le droit d'y cueillir des plantes. Le jour de la Toussaint, une troupe considérable d'hommes et de femmes ont pillé les fleurs, en menaçant de maltraiter les gardesbosquets qui voulaient s'y opposer [...]. En vain, j'ai donné des ordres de mener les délinquants à la section, les gardes n'osent les exécuter [...]. Il est cependant urgent d'obliger les mauvais citoyens à respecter les propriétés publiques " ${ }^{51}$. À la conception d'un espace pédagogique susceptible d'être ouvert sur la cité s'oppose une conception plus scientifique qui revendique une fermeture du jardin comme lieu exclusif de conservation des collections et de production scientifique. Ces débats rejaillissent avec acuité en juin 1793 lors de la création du Muséum national d'histoire naturelle. Ce que l'on peut constater, c'est que, pendant la Terreur, le Muséum s'impose comme un lieu de construction du savoir et non comme un "musée " pédagogique, conception qui prédominait pourtant au départ ${ }^{52}$. À tort, la suppression de l'Académie des sciences votée le 8 août 1793 a été considérée comme un tournant "anti-scientifique » de la Révolution. Or, ce n'est pas une provocation de dire que la période de la Terreur et du gouvernement révolutionnaire, loin d'apparaître comme une période de déclin des sciences (dont il est inutile ici de démontrer la fausseté), peut au contraire être considérée comme un moment de renforcement de la "science sévère " et d'accélération du processus de spécialisation scientifique. Les activités scientifiques de la Société d'histoire naturelle et de la Société philomathique qui servent d'espaces de retrait après la suppression de l'Académie royale des sciences, sont autant de témoignages de l'institutionnalisation de la spécialisation scientifique, renforcée, comme le souligne Roger Hahn, par la «mobilisation» des savants sous l'égide du Comité de Salut Public ${ }^{53}$. En dépit de la fin tragique de Lavoisier, la période de la Terreur marque le succès, mais plus encore, la fructification des recherches menées, sous le contrôle de l'État, par les représentants de la "science sévère ». De fait, la période 1793-1794, alors que la science est appelée à participer à l'entreprise de régénération sociale et politique, est caractérisée par une tension entre le processus de spécialisation et l'inévitable distanciation entre les savants et le "peuple » que cette spécialisation semble entraîner. La suppression de l'Académie des sciences au nom de la lutte contre les "corps » ne règlent rien puisque, parallèlement, l'État protège et encourage l'institutionnalisation et la spécialisation des savoirs au sein de "commissions» rattachées au Comité de Salut Public. À partir de 1793 et de la mobilisation de la science liée au contexte de guerre, l'organisation étatique de la recherche scientifique dans le domaine de la défense nationale et de l'industrie étudiée par Patrice Bret ${ }^{54}$ ne fait que renforcer le processus de spécialisation et de renforcement des savoirs. Si l'on peut parler d'une véritable "démocratisation de l'invention", ce processus marque néanmoins une confiscation de la science-sous contrôle de l'État- par un groupe de savants-experts considérés comme des "recours » vers lesquels se tourne l'État pour résoudre des problèmes techniques. Il y a là une véritable tension et contradiction que les élites politiques tentent de lever en attaquant la science "spéculative ", justifiant ainsi la suppression de l'Académie des sciences, et 
en promouvant le modèle de la science « utile ». Ce que l'on appelle traditionnellement "vulgarisation » durant cette période se trouve justement dans la valorisation de la science "utile ", mobilisée dans la politique de la Terreur pour répondre aux besoins militaires, industriels ou agricoles : la science (la chimie, la médecine, la physique et l'histoire naturelle), conçue comme un instrument de «bonheur», est ainsi appréhendée à travers les découvertes techniques et autres innovations technologiques susceptibles de servir aux différents membres du corps social. Les différents journaux, almanachs (pensons à l'Almanach du cultivateur de Romme), "annuaires" ou "catéchismes" rédigés à destination des habitants des villes et des campagnes témoignent parfaitement de ce phénomène. Dès l'automne 1793, des ouvrages vulgarisant les nouveaux procédés techniques furent diffusés sur ordre de la Convention, tel l'Avis aux ouvriers en fer sur la fabrication de l'acier (Berthollet, Vandermonde, Monge) ou les Procédés de la fabrication des armes blanches (Vandermonde). Or, si les «produits» de la science font l'objet d'une large diffusion (pensons encore aux expositions des objets scientifiques lors des fêtes révolutionnaires), la science, ses représentants et les modalités de sa production, tendent à se renfermer dans des lieux exclusivement ouverts aux « spécialistes » et à ceux qui, par leurs fonctions particulières (militaires, ingénieurs, industriels ou agriculteurs) sont amenés à mettre en pratique les découvertes. S'il y a bien une réaction contre une science "érudite » et fermée aux seuls savants, nous sommes loin d'une science véritablement « populaire».

Ce qu'il convient donc de considérer comme une «fermeture » de la science durant la Terreur provoque des réactions : pour certains, la science, même «utile », est moins accessible au peuple. C'est la position défendue par Henri Descremps (1746-1826) dans son ouvrage La science sans-culottisée publié en 1794, ouvrage important car il tente de construire une nouvelle conception, la "science populaire" et met en lumière des critiques contre la science que l'on retrouvera par la suite. Dénonçant l'usage d'une langue incompréhensible pour le commun, il dénonce l'inaccessibilité des ouvrages scientifiques et la distance entre la science et le «peuple » : «Les défauts essentiels des auteurs se réduisent à cinq principaux; savoir, l'obscurité du style, le défaut de méthode, l'omission des preuves et de plusieurs vérités importantes, la discussion des questions oiseuses et l'insuffisance des figures gravées, qui telles qu'elles sont, ne contribuent pas assez à l'intelligence du discours " ${ }^{55}$. Le reproche le plus important concerne le rôle "passif " dans lequel le peuple est maintenu: pour l'auteur, la "science sévère" a réduit le "peuple" en "spectateur", critique dont les enjeux politiques peuvent d'ailleurs apparaitre clairement. Dès lors, les savants tendent à remplacer les prêtres et, sous couvert de science, à envelopper le peuple d'un nouveau voile de mystère ${ }^{56}:$ « La science a-t-elle besoin, pour mériter nos respects, d'être ainsi couverte d'un voile? Les savants doivent-ils, comme les francs-maçons, employer un langage mystique, pour ne pas montrer la lumière à ceux qu'on appelle profanes? $\mathrm{Ne}$ dirait-on pas que les auteurs, comme les prédicants des fausses religions, affectent quelquefois de parler un langage inconnu au peuple, pour se dispenser de lui parler raison " ${ }^{57}$. Il s'en prend donc à ceux qui entendent « éclairer » le peuple sans lui donner réellement les possibilités de s'instruire: "Il est des auteurs élémentaires qui ont un autre défaut bien remarquable ; ils disent tout et ils ne prouvent rien ; à chaque instant, il faut les croire sur leur parole ; le lecteur après les avoir parcourus, se croit instruit, et il n'est que crédule. [...] Laissons donc là ces auteurs empiriques et subalternes qui nous étalent leur doctrine comme on prêche des mystères à des enfants, et qui dans leurs 
ouvrages, comme dans un catéchisme semblent vouloir éteindre le flambeau de la raison et l'exiger de nous qu'une imbécile crédulité " ${ }^{58}$. Cette position de Descremps n'est pas isolée. Elle est relayée par d'autres qui entendent « régénérer les mœurs » en voulant «populariser» la science. C'est par exemple le cas des rédacteurs du Journal d'instruction publique (dont le premier numéro est publié le 5 thermidor an II) qui s'attaquent autant aux "vils flatteurs des femmes " qu'aux savants qui entendent retrancher la science dans le mystère. Pour eux, «l'étude des sciences, autrefois hors de la portée du peuple, doit être simplifiée. La Physique, l'Histoire naturelle et les Mathématiques doivent être familières à tout le monde " ${ }^{59}$. On peut constater que ceux qui défendent cette "popularisation » des sciences sont souvent exclus de l'espace de la "science sévère ». Dans de nombreux cas, il s'agit d'ailleurs d'anciens partisans d'une «science mondaine » qui tente -à travers le transfert du "public éclairé » vers le peuple « sans-culottes »- de reconquérir une certaine réputation. La défense d'intérêts individuels justifie ainsi pour Descremps l'investissement dans la promotion d'une science où le peuple deviendrait un véritable acteur de la production scientifique en participant à la rédaction d'un Traité d'astronomie sans-culotisé :

«Depuis plusieurs années je travaille à faciliter l'étude de l'astronomie aux marins et aux gens de lettres. J'ai donné des leçons sur cette partie, (tant en français qu'en anglais), à des femmes, à des enfants et à des capitaines de vaisseau ; [...] pour les porter à ce degré d'instruction, il m'a souvent fallu changer la forme des explications que je trouvais dans les livres, et plus souvent encore il m'a fallu créer des démonstrations nouvelles qui pussent servir comme d'échelon et de marchepied pour parvenir aux vérités que j'avais en vue. [...] J'ai pensé que ma méthode pourrait être agréable aux curieux et utile aux marins; mais je n'ai pas voulu la faire imprimer en entier, 1. parce que j'ignore si le public pensera comme moi sur l'utilité de mon ouvrage ; 2 . parce que ma fortune ne me permet point de faire imprimer à mes frais un ouvrage de si longue haleine. Pour savoir à quoi m'en tenir sur le premier inconvénient, et pour sonder l'opinion publique, je publie ce premier Essai qui pourrait servir de préface à mon Traité d'Astronomie sansculottisée : le reste suivra de près si le public veut bien consentir à partager avec moi les frais d'impression; et pour qu'il ne soit pas leurré d'un faux espoir et victime de sa crédulité, comme dans les souscriptions ordinaires, voici ce que je propose [...]. Il y aura des chapitres consacrés à dissiper tous les nuages qui auraient pu se glisser dans les chapitres précédents. Par ce moyen, je ne serai, pour ainsi dire, chargé que de la rédaction : des morceaux précieux me seront fournis par des savants, des gens de lettres et des femmes d'esprit : mes correspondants seront les véritables auteurs, et l'ouvrage, enfant du public, ne pourra pas manquer de lui plaire.» ${ }^{60}$

11 Ces tentatives pour " démocratiser » la science et faire entrer le peuple dans l'espace des modalités de sa production et de sa validation, seront fortement attaquées après la chute de Robespierre et sous le Directoire. Si la période thermidorienne et le Directoire marquent une réaction contre la fragmentation des savoirs et tendent à réaffirmer l'idéal d'un ordre encyclopédique dont l'Institut national devient le symbole ${ }^{61}$, il y néanmoins une réelle continuité avec la période de la période de la Terreur. Parallèlement à la volonté de construire une science " encyclopédique »-l'Idéologiedont Pierre-Jean-Georges Cabanis et Antoine Destutt de Tracy posent les fondements, se met en place la conception d'une « science pédagogique ». Le savant, qui investit de nouveaux terrains d'action (à travers les fameuses "sciences morales et politiques »), devient, aux côtés du propriétaire, un modèle de citoyenneté : en échange de cette promotion politique, il se voit assigné une mission d'éducation et d'instruction. À partir de 1795 , ce que l'on entend par «vulgarisation » devient synonyme d'» enseigner » ${ }^{62}$. 
Or, cette nouvelle mission n'est pas confiée à n'importe qui : les savants pédagogues ne peuvent être choisis que parmi les savants consacrés, professeurs dans les institutions pédagogiques largement hérités de la période antérieure (École Polytechnique, École normale de l'an III, Muséum national d'histoire naturelle...) et membres de l'Institut national. La science devient en quelque sorte trop sérieuse pour que sa diffusion soit confiée à n'importe qui. Avec la remise en place des nouveaux cadres de légitimation scientifique, les savants consacrés tendent à dénoncer les "demi-savants " qui, à l'instar de Descremps, entendent construire une « science populaire ». Pour eux, le fait d'avoir voulu " populariser » la science aurait entraîné des erreurs et, par-là, provoqué la Terreur. Cette dénonciation est particulièrement évidente dans le discours de Sylvestre-François Lacroix (1765-1843), membre de l'Institut national et professeur de mathématiques à l'École centrale des Quatre Nations :

«Vouloir propager également toutes les sciences, et rendre tout le monde également instruit, est une entreprise aussi chimérique que d'amener l'égalité dans les fortunes: mais, si l'excessive richesse placée à côté du dénouement absolu, dénote un mauvais gouvernement, et tend chaque jour à en augmenter les vices, beaucoup de savants isolés chez un peuple dont la masse est plongée dans une profonde ignorance sont au moins inutiles, s'ils ne deviennent pas nuisibles en donnant crédit à quelques opinions dangereuses. Il faut que les esprits soient gradués, par rapport à la culture, comme les conditions par rapport à la fortune ; ce sont les intermédiaires qui lient les extrêmes et font la force du tout. En parlant d'intermédiaires, je suis loin de vouloir désigner par ce mot les demi-savants contre lesquels on s'est toujours élevé avec raison ; les demi-savants sont ceux qui veulent disserter sur tout, sans avoir rien approfondi, et qui sur les divers objets de nos connaissances n'ont que des idées superficielles: ils cesseraient d'être des demisavants et d'en avoir les ridicules penchants, s'ils possédaient à fond un art ou une science. Le temps que leur aurait coûté ce savoir particulier, leur rappellerait sans cesse le peu des valeurs des accessoires qu'ils auraient pu conquérir ailleurs; et ils en retireraient un profit réel dès qu'ils n'en feraient plus l'aliment d'une stérile vanité.» ${ }^{63}$

12 Comme l'illustrent de nombreuses publications, la mission pédagogique désormais assignée à la science, devient l'apanage exclusif des représentants les plus éminents de l'espace savant, représentants qui sont parallèlement investis symboliquement (à travers l'héroïsation de la figure du « savant-législateur ») d'un rôle fondamental dans l'entreprise de stabilisation sociale et politique dans laquelle le nouveau régime puise sa légitimation. Dans la configuration de cette «science pédagogique », le « public » est assimilé au "peuple-enfant» que le savant doit "guider»: il convient donc de chercher à "élémentariser » les savoirs scientifiques ${ }^{64}$, tentative menée à travers la création des écoles centrales et l'entreprise de publication de livres élémentaires ${ }^{65}$ pour laquelle les autorités sollicitent les savants les plus éminents. Or, nous savons que cette entreprise, menée à une période où les théories de Condillac sont érigées en véritables paradigmes, connaît un succès très mitigé. Ayant suivi quelques cours donné par les professeurs de l'École normale de l'an III, le député Jacques-Michel Coupé ne peut que constater le décalage et la distance entre les sciences et le "peuple»: «Personne n'estime plus que moi les rares savants que l'on a entendus à l'école normale: je vais les entendre autant que je le peux; mais ce n'était pas là qu'ils devraient être, c'était à l'académie des sciences, dans la région qui leur appartient, et de laquelle ils peuvent tout éclairer. [...] C'est en voulant scientifiser et gymnastiser ce qu'il y a de plus naturel, que l'on a cessé de bien concevoir le plan que nous avions à suivre, qu'on l'a présenté impraticable, et peut-être ridicule. Avec quel sourire le 
peuple ne regarde-t-il pas nos idées savantes $! »{ }^{66}$. En fait, comme l'a montré C. Blondel dans son étude sur les leçons données à l'École Normale de l'an III par René-Just Haüy ${ }^{67}$, le Directoire se trouve confronté à une contradiction: d'un côté, il s'agit de faire progresser les sciences en renforçant la normalisation engagée à partir des années 1780 autour de la science sévère (nosologies, taxinomies...) ; de l'autre, il faut inventer une nouvelle pédagogie, fondée sur la méthode analytique, susceptible de faire que les méthodes scientifiques (seules susceptibles de maintenir la raison dans de "justes bornes » et d'éviter le retour à la «barbarie ») soient accessibles au plus grand nombre selon «l'idéal civilisateur » défendu par le pouvoir thermidorien et directorial ${ }^{68}$. Or, l'élémentarisation des sciences se heurte justement au processus de normalisation des savoirs que l'idéal encyclopédique des «Idéologues » ne contribue pas à freiner, au contraire.

13 Face à cette normalisation qui prend assise sur la promotion d'une langue scientifique érigée en support des progrès de la Raison, Daubenton rappelle en 1798 que le savant « doit travailler pour ses compatriotes, surtout quand la nation dont on est membre favorise le progrès de la science, et indemnise les professeurs pour l'instruction publique" ${ }^{69}$. Face au demi-échec d'une "science pédagogique» confiée aux représentants les plus éminents de l'espace savant, les autorités se tournent vers d'autres acteurs qui sous le titre d'» instituteurs » (pensons à Louis-François Jauffret) ou de " pédagogues » tentent justement de construire un espace de médiation entre la science et les citoyens. À un moment où de nombreux hommes de lettres ou de sciences, situés hors des institutions intellectuelles dominantes, espèrent se tailler une réputation en investissant l'espace littéraire ou scientifique ${ }^{70}$, la "science pédagogique » constitue indéniablement une opportunité. Comme Nicolas Jolyclerc, ils se présentent en intermédiaires, en "traducteurs", capables de décrypter la langue scientifique et de ne pas se perdre dans le « labyrinthe » des sciences:

«Je n'écris pas seulement pour les savants; (je répète ici ce que je déclare à la tête de tous mes ouvrages); je n'écris pas seulement pour ceux à qui une éducation suivie a ouvert la carrière des belles-sciences et des arts utiles; je sais qu'ils peuvent se passer de moi; mes ouvrages sont adressés à toutes les classes de citoyens, à tous ceux dans qui le sentiment d'aimer et de connaître cette belle Nature, est inné comme dans moi ; ils sont dédiés à toutes les conditions d'homme ; à tous les sexes, à tous les âges; et si je ne crains pas de descendre jusqu'à des détails qui, peut-être, paraîtront minutieux; si, dans les sciences que mes écrits enseignent, je m'efforce de tout désigner, de tout interpréter, de tout simplifier, c'est que mon désir est que mes leçons soient comprises, s'il est possible, par l'enfant même qui vient de naître; c'est que je veux éviter à mes semblables les études longues et fastidieuses qui ont fatigué mon enfance et occupé tout mon premier âge [...]. J'ai percé les murs et rectifié les détours d'un terrible labyrinthe ; je résoudrai l'énigme; j'abattrai les murs et suivrai les contours du dédale; d'une science qui jusqu'ici n'était accessible qu'à un petit nombre d'individus, je parviendrai à faire une science aussi générale qu'elle est utile, aussi répandue qu'elle l'était peu; ;'en ferai une science universelle ; j'en ferai la science de tous les hommes; c'est là mon espoir; ce sera le but de tous mes travaux et de tous mes écrits.»"

14 Or, cette intrusion d'acteurs qui tentent d'ouvrir l'espace de la vulgarisation et, par-là, de la réputation, provoque une levée de la part des savants consacrés largement soutenus par les rédacteurs des journaux scientifiques tels que la Décade philosophique ou le Magasin encyclopédique. Les critiques contre les "compilateurs» et autres «charlatans » qui entendent revendiquer une compétence scientifique et s'arroger le 
statut de savant provoque en effet une offensive des savants éminents qui entendent affirmer un contrôle, aussi bien sur la production, que sur la diffusion des savoirs. Jolyclerc est lui-même victime de ces attaques, «avouant que l'auteur d'un journal estimable [le Magasin encyclopédique] m'a demandé pourquoi je prends le titre de Naturaliste, écrivant sur l'Histoire naturelle, comme si on pouvait parler de la Nature sans être Naturaliste " ${ }^{72}$. Cette réaction défensive des membres des institutions savantes provoque des réactions et de violents débats qui peuvent, à bien égards, justifier la position des membres du Portique républicain qui, en 1799, dénoncent «l'arrogance » des membres de l'Institut national et cherchent, en dehors des voies suivies par l'élémentarisation des sciences, les outils susceptibles de "régénérer » la République ${ }^{73}$. Ces tensions qui perdurent pendant les premières années du Consulat atteignent leur paroxysme en 1802, une année où plusieurs phénomènes se croisent pour provoquer d'importantes transformations dans l'organisation du monde des sciences.

III. De la professionnalisation à la vulgarisation

15 La crise économique qui touche le monde de l'édition ${ }^{74}$ conjuguée à la reconquête catholique ouvrent non seulement une période de mise en cause de la « suprématie » de la science, mais une mutation majeure de son organisation institutionnelle. Face à l'offensive menée par les porte-parole catholiques, alliés dans leur combat aux hommes de lettres soucieux de revendiquer une autonomie de la littérature face aux sciences, les scientifiques doivent prendre une position défensive. À l'instar du combat mené contre la "science sévère" dans les années 1790 , les savants et leur langage sont accusés d'avoir "dégradé l'homme » et perverti l'ordre social et politique ${ }^{75}$. Pour Chateaubriand, il ne fait aucun doute que les savants doivent être amalgamés aux "barbares" et aux "vandales " ${ }^{76}$. En France, comme de nombreux États européens, l'idée selon laquelle les sciences (et en premier lieu, la "science de l'homme»), leur diffusion et leurs progrès, ouvriraient les portes du "bonheur", est de plus en plus contestée au profit de la littérature et de la poésie. La dévalorisation symbolique de la science se traduit, par exemple, par la disparition progressive de la «poésie descriptive » qui avait joué un rôle dans sa promotion depuis la fin du XVIIIe siècle ${ }^{77}$ : la revendication d'une distinction entre sciences et lettres et l'émergence d'une "double culture », est le signe indéniable que la science perd du terrain et que l'intervention des savants sur des terrains qu'ils s'étaient arrogés (la morale par exemple) est de plus en plus contestée.

Mais ces attaques particulièrement violentes ne remettent pas en cause le statut du savant consacré et éminent. Au contraire, ces attaques vont participer au renforcement de son statut et à la légitimation de la "science officielle » qui s'impose complètement sous l'Empire. En effet, si ces attaques ont pour conséquence un retrait de la science du corps social et politique, elles ne mettent pas en cause le processus de spécialisation qui, à l'inverse, se renforce par le biais de l'intervention de l'État. La réforme de l'Institut national en 1803 signe, plus que la «défaite» des Idéologues, la faillite de l'idéal d'une « République des savants » et de l'organisation encyclopédique du monde des sciences. Dès lors, le processus de spécialisation en marche depuis les années 1780, s'accélère au sein des institutions pédagogiques, une accélération d'autant plus rapide qu'elle se double d'un processus de "professionnalisation " largement soutenu par l'administration qui entend faire de la science une véritable "institution d'État ». À travers la réforme des institutions pédagogiques en 1802, l'État favorise en effet 
l'émergence d'un groupe de professeurs qui s'érigent en "savants professionnels » et dont l'autorité est en grande partie construite sur les bénéfices financiers et symboliques concédés par l'État comme le montre la trajectoire d'un Georges Cuvier ${ }^{78}$. Revendiquant le contrôle exclusif des normes et des matériaux de production du savoir scientifique (à travers la lutte pour le contrôle des collections ou la mainmise des savants « sédentaires » sur les " voyageurs-collecteurs " ${ }^{79}$ ), les professeurs dessinent les contours d'une "science officielle» au sein de laquelle ils s'arrogent un véritable pouvoir discrétionnaire. Ils multiplient les attaques contre les "amateurs" et réinstaurent une logique de patronage qui leur permet de contrôler la production et la diffusion du savoir dont ils ont la charge. Alors que la nouvelle mission assignée aux sciences (pensons aux statistiques départementales ${ }^{80}$ ) n'est plus de "civiliser » ou d'» éduquer ", mais de contrôler les populations et de participer à la gloire du nouveau régime comme instrument de propagande, nous assistons ainsi dès le Consulat à une coupure entre la "science officielle » et le " public », une coupure qui ne cessera de se renforcer sous l'Empire ${ }^{81}$. Dans ce mouvement, les " professeurs » sont - ce n'est pas un paradoxe - soutenus par les catholiques et hommes de lettres qui dénoncent les « demisavants » et autres « instituteurs » sur lesquels retombent désormais la responsabilité des violences et désordres révolutionnaires. Ce processus constitue indéniablement une particularité française qui renvoie à la nature des rapports tissés entre l'État et la science. Le fait que les savants "professionnels » peuvent s'appuyer pour construire leur autorité sur le soutien financier de l'État renforce une coupure entre la science et le « public » qui est freiné ailleurs. Ainsi, à la même période, dans les Provinces-Unies où la structure de la recherche scientifique reste décentralisée et largement liée aux financements privés, la mathématisation des sciences et le modèle social qu'elle suppose ne parviennent pas à s'imposer. Comme le souligne Wijnand Minjnhardt, lorsque le secrétaire de la Société Hollandaise des sciences, Martinus Van Marum, prend fait et cause pour la mathématisation française, il perd ses financements privés ${ }^{82}$. En France, alors que la science - et en particulier la science mathématique - devient le socle à partir duquel se construit un véritable modèle technocratique ${ }^{83}$, l'État joue un rôle essentiel dans la coupure entre la culture scientifique et la culture des élites. Or, c'est justement parce que se constitue la « science officielle » et que s'élargit la coupure entre les savants patentés et le public que la période impériale va rendre possible l'émergence d'un espace à la "vulgarisation » scientifique qui permet la réintroduction de la science dans la "culture lettrée ». Notons d'ailleurs que c'est à cette époque que le verbe « vulgariser » fait son introduction dans le langage.

Pietro Corsi a parfaitement montré, à travers l'étude des stratégies éditoriales menées entre 1802 et 1804 par les "héritiers de Buffon" réunis autour de Charles-NicolasSigisbert Sonnini de Manoncourt, comment se constituait une résistance efficace au processus de confiscation (sociale et théorique) de la science ${ }^{84}$. Plus largement, se constitue une alliance entre les "victimes" du nouvel "ordre des sciences" regroupant les héritiers de Buffon, les médecins partisans de la «médecine philosophique " (pensons à un Jacques-Louis Moreau de la Sarthe qui mène le combat dans les colonnes de la Décade), mais aussi les défenseurs de la statistique descriptive réunis un moment autour de Louis-Joseph-Philippe Ballois et des Annales de statistique qui s'opposent aux partisans de la mathématisation ainsi que les promoteurs du galvanisme ${ }^{85}$. En dépit de la crise éditoriale, ils parviennent, souvent en se regroupant, à diriger des entreprises efficaces à travers lesquelles ils s'opposent aux normes théoriques et aux règles sociales de la "science officielle " ${ }^{86}$. Au moment où certains 
éditeurs choisissent - et par là-même contribuent à renforcer - une stratégie de spécialisation et de professionnalisation (c'est le cas de l'éditeur Levrault qui, avec la collaboration des professeurs du Muséum, lance en 1802 ses Annales du Muséum), d'autres, en collaboration avec les opposants à la "science officielle ", s'engagent dans des productions destinées au «large» public. En 1798, à l'initiative de l'imprimeur Dufart et en collaboration avec le jeune médecin Virey, Sonnini dirige la réédition de l'Histoire naturelle de Buffon (1798-1808; 127 vol.); quelques années plus tard, en octobre 1802, Sonnini et l'imprimeur-libraire F. Buisson ${ }^{87}$ relancent la publication de la Bibliothèque physico-économique ${ }^{88}$ qui avait cessé en 1795 ; la même année, Sonnini dont l'activité, on le constate, est particulièrement dynamique ${ }^{89}$, s'engage, avec l'imprimeur Déterville, dans l'édition du Nouveau Dictionnaire d'histoire naturelle (1802-1804, 24 vol.). Ouvrant la voie à une véritable " bataille des dictionnaires », ces publications font, dès leur annonce, l'objet des plus vives attaques de la part des savants consacrés, en particulier de Georges Cuvier qui, au même moment, est chargé, par l'éditeur Levrault, de diriger l'édition du Dictionnaire des sciences naturelles. Cuvier accuse les «libraires » de "spéculations commerciales » et s'attaque aux compilateurs, ceux qui « retirés dans leurs cabinets, seulement avec des livres, renonçant à l'observation, dénués même pour la plupart des moyens d'observer, [...] ont cru enrichir le système de la nature en remplissant ce vaste catalogue de phrases recueillies de toutes parts, sans comparaison, sans examen des autorités dont elles provenaient et en les accompagnant d'une foule de citations discordantes et souvent contradictoires" ${ }^{90}$. Dans cette lutte où se juxtaposent intérêts commerciaux et scientifiques, Sonnini réplique immédiatement en mettant en cause la position du « professeur »[⿷匚⿳0口䒑0: « je ne sais quel pédagogue [...] soutint qu'à lui seul appartenait le droit de parler d'histoire naturelle. Les sciences sont-elles donc matière à privilèges exclusifs ; et parce qu'on est payé pour les enseigner, sera-t-il défendu de s'en occuper à d'autres qui ne le sont pas? " ${ }^{91}$. Reprenant des arguments anciens, Sonnini et ses alliés (en particulier Virey et Denys de Montfort) dénoncent la "scolastique » dont se pare les tenants de la «science officielle» amalgamés à une " secte ", et revendiquent une science accessible au plus large public:

«Par quelle fatalité cette science est-elle devenue au contraire un assemblage de systèmes, de méthodes, de discussions de nomenclature aussi sèches qu'oiseuses [...] ? Quand enfin verrons-nous tarir ce torrent bourbeux de mots nouveaux et barbares, qui inonde presque tous les livres modernes d'histoire naturelle, rend la langue de la science plus embarrassante et plus difficile que la science elle-même, et nous menace de nous faire retomber aux siècles du jargon scolastique, compagnon fidèle de l'ignorance et des ténèbres ? [...] Il est facile de concevoir que des ouvrages composés dans un pareil esprit n'offrent pas même d'intérêt du côté du style et des accessoires; tout ce qui montre quelque apparence d'agrément en est rigoureusement repoussé ; non seulement on n'y attache point de prix à la manière d'écrire, mais celle qui n'est ni dure ni sèche, ni barbare, en est impitoyablement proscrite; un jargon énigmatique, que les initiés même ont beaucoup de peine à comprendre est exclusivement employé ; ce sont des termes consacrés, auxquels il est interdit d'en substituer de plus intelligibles; ce serait une profanation que d'y en adjoindre qui fussent d'un usage général [...] et ce n'est pas un travail de peu de conséquence de parvenir à comprendre un langage fort mal à propos appelé scientifique, et à se ranger au nombre de ses adeptes [...] c'est ce qu'ils nomment le style sévère : et il faut convenir, une pareille sévérité devient redoutable à toutes les classes de lecteurs [...]. L'intérêt des sciences exigerait, ce me semble, que de savants si profonds, mais en même temps incompréhensibles, renonçassent, du moins pour quelques temps, à la gloire de créer des mots et même d'enfanter des systèmes, pour observer, recueillir des faits ou les vérifier [...]. Les sciences sont- 
elles matière à privilèges exclusifs ; et parce qu'on est payé pour les enseigner, serat-il défendu de s'en occuper à d'autres qui ne le sont pas? Le public éclairé ne fait aucune différence entre le savant en place et le savant isolé, et il ne les juge que d'après leurs productions et nullement d'après leur morgue ou leurs prétentions présomptueuses. J'écarterai principalement toute dénomination grecque qu'un long usage n'aura pas consacré ; et dussé-je passer aux yeux de certains naturalistes de nos jours pour un esprit vulgaire, je parlerai français, et leur laisserai volontiers la prérogative, très relevée sans doute, de parler sans être entendus. [...] Assez d'autres s'environnent de ténèbres en faisant usage d'une multitude de mots inintelligibles; je m'efforcerai à me rendre clair, et c'est, à mon sens, le seul moyen de faire aimer l'étude de la Nature, et d'y disposer un plus grand nombre de personnes.» ${ }^{92}$

18 En rééditant les œuvres de Buffon, de Vicq d'Azyr ou de Lavater, les opposants à la "science officielle» ne défendent pas une position nostalgique dont le caractère hétéroclite des héritages marquerait, face aux progrès d'une science "moderne ", la nature passéiste et archaïque. En prenant partie pour le «beau style » susceptible de toucher un public plus large que les seuls spécialistes ${ }^{93}$, ils entendent s'ouvrir un espace que l'on peut définir comme celui de la science "utile ». La publication en 1808 de la liste des souscripteurs de l'Histoire naturelle (753 noms) nous permet de saisir à peu mieux le « public » de cette science « utile» :

Répartition socio-professionnelle des souscripteurs de la réédition de l'Histoire Naturelle de Buffon (1798-1808) chez Dufart ${ }^{94}$

\section{Voir document annexe}

Loin d'être des "marginaux » soucieux de se tailler une réputation dans le combat mené contre les savants consacrés, les opposants à la « science officielle » entendent s'inscrire dans l'héritage de la voie ouverte à la fin du XVIIIe siècle et élargie pendant la Terreur, à savoir une science ouverte vers l'industrie, l'agriculture et l'armée, une science de l'ingénieur. Le retrait de la science spéculative à l'intérieur d'institutions qui se constituent en espaces exclusifs et fermés, se met en place une science ouverte sur la société qui s'institutionnalise, autour d'un Chaptal ou d'un Parmentier par exemple (protecteur de Sonnini), au sein de la Société d'agriculture ou de la Société d'Encouragement pour l'industrie nationale. On peut constater que les opposants de la «science officielle » sous l'Empire ont été, entre 1793 et 1794 et aux côtés d'un Gilbert Romme et de son Annuaire du cultivateur, des personnalités particulièrement actives dans la diffusion des techniques et des produits de la science. S'ils semblent récupérer l'héritage de la «science mondaine " ${ }^{95}$, ils cherchent surtout à construire et à institutionnaliser les frontières d'un espace nouveau, celui de la "science utile ", au sein duquel les exclus de la « science officielle » auraient leur place ${ }^{96}$. Entre 1808-1810, période pendant laquelle les fameux «rapports " à l'Empereur sur les progrès des sciences, des lettres et des arts institutionnalisent les nouvelles normes imposées par l'État à l'organisation disciplinaire et institutionnelles des différentes productions intellectuelles ${ }^{97}$, les ouvrages des opposants à la " science officielle » rencontrent un succès commercial important auprès des nouvelles élites sociales et politique du Consulat et de l'Empire. Or, parallèlement à l'ouverture et à l'institutionnalisation d'un espace de la science « utile » (à travers la création de la Société pour l'Encouragement de l'industrie nationale en 1801 et de la Société d'agriculture en 1802) qui parvient progressivement à s'imposer aux côtés de la « science officielle » (et dans une certaine mesure intègre l'espace de la «science officielle » qui devient un champ de tension 
entre les « savants » et les «ingénieurs »), s'ouvre sous l'Empire, un troisième espace, celui que l'on peut qualifier sous le nom «vulgarisation scientifique». Si les publications scientifiques spécialisées rencontrent des difficultés financières et commerciales du fait du caractère restreint du public auquel elles s'adressent, le succès commercial des ouvrages agronomiques ou techniques montre qu'il existe un public pour la science hors du groupe des professeurs et de leurs élèves. Dans le prospectus de la Bibliothèque physico-agronomique, Sonnini trace les contours de ce public composé d'» administrateurs, propriétaires, agronomes, cultivateurs, curés, physiciens, savants, artistes, manufacturiers ", un public choisi dont les membres se retrouvent parmi le personnel des sociétés savantes de province sous l'Empire ${ }^{98}$. Or, aux côtés des destinataires de cette science utile, se reconstituent les contours d'un public «mondain» dont la culture lettrée s'ouvre à la science. C'est ce public composé des élites sociales qui va être le support de la «vulgarisation scientifique » et de son autonomisation progressive au XIXe siècle. Cette «vulgarisation » qui se constitue au moment où s'affirme la «science officielle " n'est pas un simple retour à la "science mondaine » : bien que le public visé soit principalement le public des élites culturelles et sociales qui se reconstitue dans des espaces de mondanité ${ }^{99}$, la "vulgarisation » ne repose plus ici (comme c'était le cas pour la «science mondaine » du XVIIIe siècle) sur une conception d'un public susceptible de participer à la production et à la validation $\mathrm{du}$ discours scientifique. De ce fait, la «vulgarisation » n'apparaît pas comme un " sous "-discours ou un contre-discours face à la "science officielle", spéculative ou utile, mais comme un discours parallèle qui se construit dans d'autres lieux, selon d'autres discours et d'autres pratiques. Cette vulgarisation n'est donc pas "dangereuse " pour les représentants de la science officielle car elle ne prétend pas faire concurrence à la science «qui se fait ». C'est donc sous l'Empire, au moment où commence l'aventure «scientiste» que les conditions de possibilités de la " vulgarisation » sont réunies : son émergence vient combler, en quelque sorte, l'espace (de la «curiosité » et de la "science agréable ») laissé par les représentants de la "science officielle ». Signe évident de la constitution de cet espace de la vulgarisation fondé sur un nouveau partage des tâches, l'essor de genres de production liés à des pratiques qui tendent à construire une nouvelle relation entre la science et le public mondain. C'est par exemple le cas des "promenades » au jardin du Muséum d'histoire naturelle qui, comme nous l'avons rappelé, avait été l'objet de tensions. Sous l'Empire, des «vulgarisateurs» proposent des itinéraires d'une promenade qui se présente comme une alternative entre l'occupation "sauvage» et la pratique cognitive du jardin. Bénéficiant d'un certain succès commercial, nous assistons ainsi à l'affirmation de nouveaux acteurs, des « vulgarisateurs » qui ne prétendent pas faire de l'ombre aux savants consacrés. C'est par exemple le cas d'un Jean-Baptiste Pujoulx (1762-1821), auteur de différents guides de "promenades » dans les jardins du Muséum d'Histoire naturelle. Il s'agit pour lui de donner au « public » la possibilité de visiter cet espace de manière « agréable » et « instructive » :

«Il faut donc que le lecteur se persuade que j'agis avec lui comme j'ai agi plusieurs fois avec des amis, qui sachant que j'aime l'histoire naturelle, m'ont engagé à les conduire, soit au jardin, soit dans les galeries, soit enfin dans les différentes parties de ces précieux établissement ; où, tout en nous promenant, je cherchais à fixer leur attention sur les plantes, les minéraux, les animaux vivants ou conservés qui me paraissent dignes de quelques remarques particulières. On sent que ce n'est ici ni un ouvrage de littérature, ni un livre de science ; il peut bien être de quelque utilité pour les littérateurs qui ne sont pas naturalistes; mais certainement il ne sera lu 
par les savants, auxquels il n'apprendrait rien ; car, je le répète, mon but n'a été que de le rendre agréable et instructif pour les gens du monde des deux sexes, et de tout âge, qui veulent tirer un peu de fruit de leurs visites au Jardin des Plantes, à la Ménagerie et aux Galeries du Muséum.» 100

21 Un autre signe de l'émergence de l'espace de la « vulgarisation » scientifique apparaît à travers la vogue et le succès commercial rencontrés par les «dictionnaires » qui prétendent fournir des connaissances scientifiques de manière la plus rapide et facile possible, connaissances susceptibles de servir de matières aux conversations des nouveaux salons mondains :

«Depuis quelques temps les Dictionnaires semblent devenir à la mode : ce sont des bibliothèques abrégées qui flattent tout à la fois le désir d'apprendre et la répugnance à travailler. Tout le monde veut paraître savant, et peu de gens veulent le devenir, si ce n'est à peu de frais, et en s'amusant par des lectures agréables qui remplissent l'intervalle des affaires et des plaisirs : on n'a garde de pousser jusqu'à la fatigue une étude qu'on ne choisit que pour se délasser. Les Dictionnaires sont d'un merveilleux secours aux personnes ainsi disposées; par le moyen de l'ordre alphabétique, on trouve recueilli dans un seul livre ce qu'il faudrait tirer, avec des recherches pénibles, d'une infinité de volumes, qu'un particulier ne saurait amasser, et qu'il serait difficile de rencontrer tous dans les plus riches bibliothèques. Avec un Dictionnaire, sans avoir rien appris, on sait tout à mesure qu'on en a besoin.» 101

Progressivement, le vulgarisateur revendique une légitimité propre fondée sur sa fonction de médiation entre le grand public et des scientifiques enfermés dans un discours ésotérique. On peut ainsi constater, dans la continuité de Claude Blanckaert, que la vulgarisation consacre, et ce n'est qu'un paradoxe apparent, l'écart entre le " public » et la science " professionnelle » ${ }^{102}$. En 1808, Saint-Simon expose une théorie de la «double vérité » qui souligne cette distanciation qui ne cessera de s'étendre au XIXe[20 idées, je ne les communique qu'aux personnes suffisamment éclairées pour envisager les choses de ce point de vue [...] Je crois que la force des choses veut qu'il y ait deux doctrines distinctes : le physicisme pour les gens instruits, et le déisme pour la classe ignorante " ${ }^{103}$. Ce que Saint-Simon ne dit pas, c'est qu'entre le "physicisme » et le "déisme ", existe désormais un espace de la "vulgarisation scientifique » qui ne cessera de s'élargir pendant tout le XIXe siècle à mesure que la fonction sociale et politique de la science sera valorisée et que les œuvres de vulgarisation cesseront progressivement d'être l'apanage exclusif des « Bouvard et Pécuchet ».

\section{ANNEXES}

" Je dois un témoignage public de reconnaissance à MM. les souscripteurs de cette édition de l'Histoire naturelle [...] qui m'ont constamment soutenu dans cette grande entreprise, pendant l'espace de huit à neuf années. Je désirerais pouvoir rendre ce témoignage digne du sentiment qui me l'inspire, et mériter, dans l'exercice de mon état, la continuité d'une bienveillance aussi honorable. " ${ }^{104}$ 
La publication en 1808 de la liste des souscripteurs de la nouvelle édition de l'Histoire naturelle constitue une étape importante dans la lutte que mène Sonnini de Manoncourt contre les « professeurs » du Muséum. Elle tend à rendre visible le succès d'une entreprise scientifique et commerciale particulièrement ambitieuse (127 volumes ont été publiés au lieu des 60 annoncés en 1798) et risquée, au moment où la publication du Dictionnaire des sciences naturelles dirigé par Cuvier associé à l'imprimeurlibraire Levrault a du être suspendu (en 1804, date de la faillite de l'imprimerie Levrault, seuls deux volumes de ce dictionnaire ont été publiés). Si elle permet de remercier ceux qui l'ont soutenu, cette liste donne cohérence à un groupe qui n'était pas construit d'avance et auquel Sonnini doit, non seulement le succès de l'ouvrage, mais aussi la légitimité de son statut de savant.

" [...] si la postérité s'empare avec gloire des hommes privilégiés d'un génie supérieur, pour éclairer et embellir la vie des hommes laborieux et honorables de la société, les descendants de ceux-ci, ne lisent pas avec moins d'admiration leurs noms, ou celui de ceux qui ont si puissamment contribué, par le fruit de leurs travaux, aux progrès des sciences exactes et à l'exécution de ces monuments du génie, qui transmettent la science aux siècles les plus reculés. »

Stigmatisé comme un « charlatan » par Cuvier, Sonnini revendique la possibilité de construire et de diffuser l'histoire naturelle en dehors des frontières du public restreint des spécialistes. Avec un total de 760 souscripteurs, ce sont près de 1350 exemplaires qui ont été vendus à Paris (685 exemplaires) et en province, les libraires (105) souscrivant le plus souvent pour plusieurs exemplaires. Cette liste permet de préciser la composition socio-professionnelle du public de la science " utile », ces nouveaux « experts » du régime impérial (membres de l'administration -43-, représentants du monde de la justice -41-, des affaires -78-, propriétaires -24 -, rentiers - 25 - et médecins $-52-. .$.$) que l'on peut retrouver parmi les membres de la Société pour l'encouragement$ de l'industrie nationale ou les sociétés d'agriculture recomposées à partir de 1802. À l'inverse, on peut constater l'absence, parmi les souscripteurs, des professeurs du Muséum d'histoire naturelle : leur spécialité n'étant pas indiquée, seuls 7 souscripteurs sont présentés comme " professeurs » et " étudiants ", signe de la distinction des publics de la science naturelle qui se formalise sous l'Empire. Comme le souligne la composition sociale du groupe important des souscripteurs rouennais, le public de l'Histoire naturelle de Buffon constitue un groupe particulièrement hétérogène ; membres de l'élite sociale, on en retrouve la plupart au sein de l'Académie des Sciences et Arts et de la Société libre pour le Commerce et l'Industrie.

Les souscripteurs de Rouen (région exclue : 132) 


\begin{tabular}{|l|l|}
\hline J.C. ALLARD, Négociant & HUE, Libraire \\
\hline ANGERVILLE, du Conseil de la Préfecture & HUET, Rentier \\
\hline ARCHAMBAULT, Négociant & JOBEY, Banquier \\
\hline ARMANDTISSON, Négociant & JUVEL, Négociant \\
\hline ARVILLE & LAMAUVE, Officier de santé \\
\hline AVIAT, Receveur des contributions & LAMBERT, Directeur de la monnaie \\
\hline BARBIER, Directeur des postes & LANELLE, Négociant \\
\hline BARDEL, Négociant & LAPALU, Rentier \\
\hline J.G. BARRE, Négociant & LEBLOND, Marchand bijoutier \\
\hline BAUDRY, Imprimeur & LEBOUCHE, Médecin \\
\hline BEAUDOIN & LEBOULLENGER, Ingénieur \\
\hline BERTRAND, Négociant & LEBRET, Rentier \\
\hline BIDAULT-MILLION, Négociant & LHERMITTE, Négociant \\
\hline BIENVENU-DUBUC, Rentier & LEPAINTEUR \\
\hline BOETE, Architecte & LEROUXEL \\
\hline BORME, Directeur du théâtre des arts & LESGUILLIEZ, Négociant \\
\hline BOUFFEY, docteur en médecine & LEZURIER jeune, Négociant \\
\hline BOULOT, chirurgien & LEZURIER laîné, Négociant \\
\hline BOURGEOIS-BONNET, Négociant & LOISELIER \\
\hline A. BROUARD, Courtier & MACE, Négociant \\
\hline BUGUET, Rentier & MAILLE, Négociant \\
\hline CAGNION & MALLEUX, Chirurgien \\
\hline CALANGE, Homme de loi & MARCHAND, Négociant \\
\hline CARBONIER, Avocat & MARTIN frères, Négociant \\
\hline CARON, Négociant & MERY, Négociant \\
\hline CHAMBOSSE, Négociant & MIDY-du-LIEUBRE \\
\hline CHESNEAU, Marchand-papetier & MIDY-DESMESNIL, Négociant \\
\hline COLONGE, Notaire & MILLEVILLE, Rentier \\
\hline J.B. CUVELIER, Avocat & MONGENDRE, Rentier \\
\hline DEFONTENAY, Négociant & MORIN, Négociant \\
\hline DELAMARRE, Juge & MULLOT, Docteur en chirugie \\
\hline DELANNOY, Négociant & NOUFFLARD, Banquier \\
\hline DELAVIGNE, Avocat & PAPILLON \\
\hline DELEAU, Architecte & PELON, Chirurgien \\
\hline DESALEURS, Médecin & PILORE, Médecin \\
\hline DESCAMPS, Peintre & PILORE, Chirurgien \\
\hline DEVIEUX, Rentier & PINEL fils, Négociant \\
\hline & \\
\hline
\end{tabular}

\begin{tabular}{|c|c|}
\hline DHONDEMARRE, Rentier & PLOUGOULM, Maître de pension \\
\hline Mme D'HYBOUVILLE & PORLIER, Chef du bureau militaire \\
\hline DUFFAY, Rentier & PORTRAIT, Homme de loi \\
\hline DUFRESNE, Négociant & POULLAIN, Huissier à la Cour \\
\hline DUPAS & PRETEL \\
\hline DUPUTEL, Homme de lettres & OUENLIN, Homme d'affaires \\
\hline DUSSEAUX, Maitre de pension & RAIMBERT \\
\hline DUVERGIER, Négociant & RECULARD, Notaire \\
\hline FAUCON, Maître de pension & RENAULT, Libraire \\
\hline FLEURY, Chirurgien & RICARD, Négociant \\
\hline FONTAINE, Architecte & ROBERT, Homme de loi \\
\hline FOULLON, Négociant & ROBERT, Pharmacien \\
\hline FRERE, Libraire & ROULLAUD, Rentier \\
\hline GABORY, Fabricant d'indiennes & ROUSSEL, Médecin \\
\hline GAILLARD, Rentier & SAUTELET, Négociant \\
\hline GARGEY, Négociant & SELLIER, Négociant \\
\hline GERMINI, Rentier & SENECHAL Négociant \\
\hline GOSSELIN, Négociant & SENEGON, \\
\hline GRANDIN, Négociant & Mme SOULES, Rentière \\
\hline GRANGER, Directeur du Thêtre des arts & SOYER, Négociant \\
\hline GRENIER, Rentier & TERNANTE, Médecin \\
\hline GRENIER DERNEMONT, Rentier & TOPNOT, Négociant \\
\hline GRESSENT, Rentier & TOUSTAIN-de-LIMESY, Rentier \\
\hline GROULT, Négociant & TREHET, Médecin \\
\hline HARDY, Chirurgien & TROUVE l'aîné, Homme d'affaire \\
\hline HEBERT, Commerçant & TROUVE le jeune, Huissier \\
\hline HELLOT, Négociant & VALLEE frères, Libraire \\
\hline HERON d'ANGIRONE, Avocat & VASSE, Négociant \\
\hline HERPIN, Avocat & VILLETTE \\
\hline
\end{tabular}

Pour des raisons techniques, il a été impossible de cartographier la présence des 39 souscripteurs présents hors des frontières de la France « hexagonale », mais dont la majorité se trouve dans des territoires appartenant au « Grand Empire » : Breslau (1), Bruxelles (3), Cologne (2), Hambourg (8), Ile de France (1), Liège (3), Madrid (13), Mons (1), Moscou (1), Naples (1), Rome (1), Saint-Pétersbourg, Vienne (2). Si, pour 17 souscripteurs, les origines géographiques ne sont pas indiquées, cette liste permet de 
mesurer d'importants contrastes dans la répartition du public de l'Histoire naturelle. Les souscripteurs se répartissent dans 74 villes ou leurs alentours (les lieux situés en zone rurale ou les villages périphériques ayant été rattachés à la ville la plus proche, la carte indiquant ainsi plus une diffusion régionale de l'ouvrage). Au-delà de la domination écrasante de Paris (394), on peut constater une concentration des souscripteurs dans les régions situées au nord de la Loire avec une nette prédominance de la Normandie (Rouen, Caen), de la Somme, de la Picardie et du Nord (Lille, Douay, Cambrai). Avec 139 souscripteurs, Rouen est la capitale provinciale où l'ouvrage s'est le plus diffusé, laissant loin derrière les autres villes : Angers (11), Caen (10), Douay (15), Lille (5), Limoges (24), Lyon (3), Nantes (5), Strasbourg (7) et Toulouse (6). Ces contrastes doivent néanmoins être considérés avec précaution. En effet, d'un côté, certains libraires de province n'ont pas fourni la liste des souscripteurs, de l'autre, nous avons construit notre carte à partir du nombre des souscripteurs et non des exemplaires souscrits, choix qui a eu pour conséquence de minorer le poids de certaines villes, particulièrement dans le sud de la Loire, constatation qui oblige à relativiser la forte domination de la France du Nord.

\begin{tabular}{|l|l|l|}
\hline & Souscripteurs & Nombre d'ex. \\
\hline \hline Bayonne & 2 & 18 \\
\hline \hline Bordeaux & 2 & 14 \\
\hline \hline La Rochelle & 2 & 14 \\
\hline \hline Livourne & 1 & 25 \\
\hline \hline Lyon & 3 & 24 \\
\hline \hline Montpellier & 1 & 54 \\
\hline
\end{tabular}

Avec les précautions d'usage, si l'on compare la géographie des souscripteurs de l'Encyclopédie in-quarto de Panckoucke et Duplain (1777-1779) présentée par R. Darnton (L'aventure de l'Encyclopédie, Seuil, 1992, p. 303) à celle de l'Histoire naturelle de Buffon en sous l'Empire, on peut constater certaines différences, en particulier dans la baisse du poids des anciennes villes parlementaires (Besançon, Rennes, Aix) qui constituaient des pôles importants de diffusion de l'Encyclopédie. La faible diffusion dans certaines régions (Ouest armoricain, Massif Central, Savoie, Pyrénées) constitue, à l'inverse, un élément de continuité. Expliquer les raisons des différences (parfois importantes) de la diffusion de l'Histoire naturelle dans les différentes villes mériterait évidemment une étude exhaustive. Une des raisons du succès de cette diffusion renvoie sans doute à la plus forte densité des librairies dans plusieurs villes (en particulier à Caen, Lille ou Douay) et au dynamisme commercial des libraires qui jouent un rôle essentiel dans les stratégies d'invention du public et, plus généralement, dans les transformations de l'espace scientifique. Cette constatation se vérifie dans le cas des frères Vallée, libraires à Rouen installés rue Beffroi, dont le rayonnement s'étend à toute la Normandie et dont l'efficacité à gagner et à fidéliser leurs clients expliquent assurément le poids 
important de Rouen et de sa région. Une autre explication peut être recherchée du côté de la sociabilité savante provinciale dont on connaît le dynamisme depuis le Directoire. La carte des sociétés savantes en 1810 présentée par J.-P. Chaline (p. 50) reproduit en effet la répartition des souscripteurs de l'Histoire naturelle. Alors que l'on assiste à une accélération du processus de spécialisation scientifique au sein des institutions scientifiques, c'est auprès de ceux qui revendiquent désormais le statut d' " amateurs » que Sonnini trouve le public de la science « utile » et plaisante.

Répartition géographique des souscripteurs de l'Histoire naturelle de Buffon (1808)
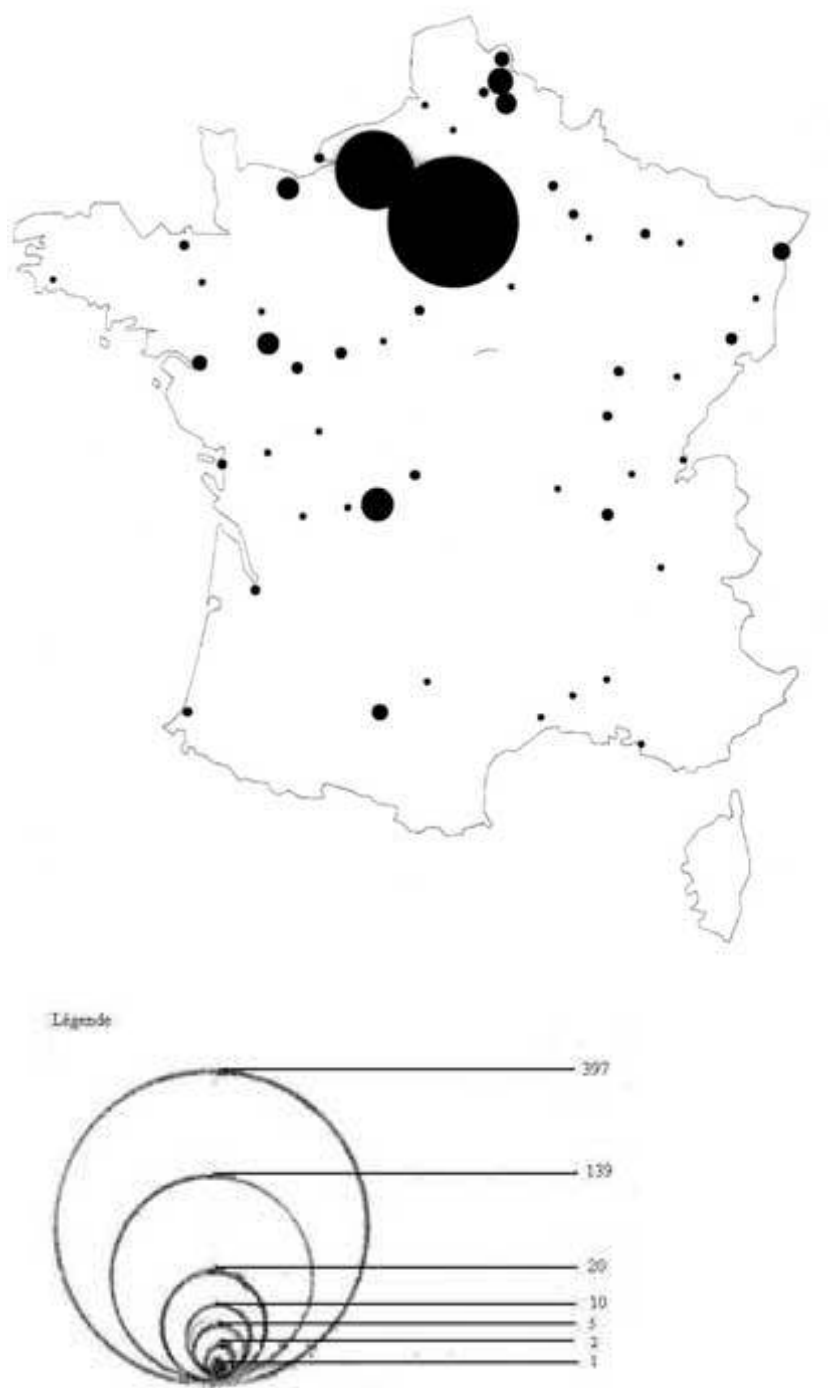

Source : «Liste de MM. Les souscripteurs ", Tables analytiques et raisonnées des matières et des auteurs pour la nouvelle édition de l'Histoire Naturelle de Buffon rédigéé par C.S.Sonnini, Paris, 1808, t. CXXVII, p. 343-355 (B.N., S 10 271).

\section{NOTES}

1.Catherine DUPRAT, « Lieux et temps de l'acculturation politique », Annales historiques de la Révolution française, 1994, n² 297, pp. 387-400. 
2.Cf. Bruno BEGUET (dir.), La science pour tous. Sur la vulgarisation scientifique en France de 1850 à 1914, Paris, Bibliothèque du C.N.A.M., 1990, 168 p.

3.Hélène METZGER, « La littérature scientifique française au XVIIIe siècle », Archeion, 1934 (16), p. 11.

4.Pour Andréas KLEINERT, " la science des "vulgarisateurs" était souvent loin d'être à la hauteur de celle des savants, et les idées que les lecteurs de ces livres [de vulgarisation] ont dû se faire sur la méthode et sur les buts de la science de leur époque correspondaient d'autant moins aux concepts des chercheurs que le siècle avançait ", « La science qui se vulgarise, la science qui se fait », in Claude BLANCKAERT et alii (dir.), Nature, Histoire, Société. Essai en hommage à Jacques Roger, Paris, Klincksieck, 1995, p. 326. Les positions de Kleinert et de Metzger sont en fait conciliables : elles renvoient à deux « moments » particuliers de la production scientifique au XVIIIe siècle.

5.Cf. par exemple, la Mathématique universelle abrégée à la portée de tous et à l'usage de tout le monde [1728] du Père Castel, du Père Regnault (Entretiens physiques d'Ariste et d'Eudoxe ou physique nouvelle en dialogues [1729] du Père Regnault, le Spectacle de la nature [1732] de l'abbé Pluche, Le Newtonianisme pour les dames [1737] d'Algarotti, ou les Études de la Nature [1787] de Bernardin de Saint-Pierre.

6.Bruno JAMMES, « Le livre de science », in Roger CHARTIER et Henri-Jean MARTIN (dir.), Histoire de l'édition française, Paris, Fayard, 1990, vol. 2, p. 256.

7.Vincenzo FERRONE, «L'homme de science », in Michel VOVELLE (dir.), L'homme des Lumières, Paris, Seuil, 1996, pp. 229-230.

8.Sur cette notion d'» amateurs » au XVIIIe siècle, voir Jean-Louis JAM (dir.), Les divertissements utiles des amateurs au XVIIIe siècle, Clermont-Ferrand, Presses Universitaires Blaise Pascal, 2000, 218 p.

9.Jeffrey S. RAVEL, The Contested Parterre. Public Theater and French Political Culture (1680-1791), Ithaca, New York, London Cornell University Press, 1999, 256 p.

10.Thomas CROW, La peinture et son public à Paris au XVIIIe siècle, Paris, Macula, 2000, $335 \mathrm{p}$.

11. Comme le souligne Daniel JACOBI, « il n'y a pas d'un côté un discours scientifique source, discours incompréhensible par le public moyen et de l'autre un discours second, reformulation et paraphrase du premier destiné au plus grand nombre, mais un continuum dans lequel les scripteurs, leurs textes et leurs diverses intentions se mêlent intimement ", Diffusion et vulgarisation. Itinéraires du texte scientifique, Paris, Les Belles Lettres, 1986, p. 22.

12.Roger CHARTIER, Les origines culturelles de la Révolution française, Paris, Seuil, 1990, chapitre 2 ; Sarah MAZA, Vies privées, affaires publiques. Les causes célèbres de la France prérévolutionnaire, Paris, Fayard, 1997, 384 p.

13.Pierre BOURLIEU, Science de la science et réflexivité, Paris, Raisons d'agir, 2001, 237 p.

14. Steven SHAPIN, Simon SCHAFFER, Léviathan et la pompe à air. Hobbes et Boyle entre science et politique, [1985], Paris, La Découverte, 1993, 457 p. ; voir aussi Jan GOLINSKI, Science as Public Culture. Chemistry and Enlightment in Britain, 1760-1820, Cambridge, Cambridge University Press, 1992, 342 p. ; J. GASCOIGNE, Joseph Banks and the English Enlightenment. Useful Knowledge and Polite Culture, Cambridge, 1994.

15. Christian LICOPPE, La formation de la pratique scientifique. Le discours de l'expérience en France et en Angleterre (1630-1820), Paris, La Découverte, 1996, 346 p. 
16. Marie-Françoise MORTUREUX, La formation et le fonctionnement d'un discours de la vulgarisation scientifique au XVIIIe siècle à travers l'œuvre de Fontenelle, Lille, Atelier national de reproduction des thèses, Paris, Didier, 1983, 731 p.

17.Christian LICOPPE, La formation de la pratique scientifique..., op. cit. Voir également Robin E. RIDER, « El experimento como espectáculo », in Javier ORDONEZ, Alberto ELENA (dir.), La ciencia y su público. Perspectivas historicas, Madrid, 1990, pp. 113-146.

18.Je remercie Jean Ehrard et Anne-Marie Bourdin pour les renseignements fournis sur cette correspondance en cours de publication.

19.Antoine LILTI, « Le monde des salons. La sociabilité mondaine à Paris dans la seconde moitié du XVIIIe siècle », Thèse d'histoire soutenue en juin 2003, Université Paris I, D. Roche dir. « Lettre de Gilbert Romme à Gabriel Dubreul », mars 1779.

20.« Lettre de Gilbert Romme à Gabriel Dubreul », mars 1779.

21.Alessandro GALANTE GARRONE, Gilbert Romme. Histoire d'un révolutionnaire (1750-1795) [1959], Paris, Flammarion, 1971, 493 p.

22.Louis-Bernard GUYTON DE MOREAU, «Sur les dénominations chimiques, la nécessité d'en perfectionner le système, et les règles pour y parvenir », Observations et mémoires sur la physique, 1782, 19, pp. 370-382 ; Louis-Bernard GUYTON DE MOREAU, Antoine-Laurent LAVOISIER, Antoine-François FOURCROY, Méthode de nomenclature chimique [...] on y a joint un nouveau système de caractères chimiques adaptés à cette nomenclature, par MM. Hassenfratz et Adet, Paris, Cuchet, 1787 : « Il est temps de débarasser la chimie des obstacles de toute espèce qui retardent ses progrès [...] c'était par le perfectionnement du langage que cette réforme devait être opérée ".

23.Antoine-Laurent LAVOISIER, Essai de nomenclature chimique, Paris, 1787.

24.Félix VICQ D’AzYR, Traité d'anatomie comparée et de physiologie, Paris, s.n., 1786, p. 1. 25.Christian LICOPPE, La formation de la pratique scientifique..., op. cit., chapitre 7.

26.Pascal DURIS, Linné et la France : 1780-1850, Genève, Droz, 1993, 281 p.

27.Cit. dans Jonathan MANDELBAUM, « Science and Friendship : The Société Philomathique de Paris, 1788-1815 », History an Technology, 1988, vol. 5, p. 180.

28. Yves GINGRAS, « Mathématisation et exclusion, socioanalyse de la formation des cités savantes », in Jean-Jacques WUNENBURGER [coord.], Gaston Bachelard et l'épistémologie française, Paris, P.U.F., 2003.

29.Eric BRIAN, La mesure de l'État. Administrateurs et géomètres au XVIIIe siècle, Paris, Albin Michel, 1994, 462 p.

30.Comme peut l'illustrer la ligne rédactionnelle suivie par la nouvelle équipe de savants réunie autour de l'Encyclopédie méthodique qui rompt avec l'ordre des savoirs suivi par les rédacteurs de l'Encyclopédie de Le Breton. Cf. Robert DARNTON, L'aventure de l'Encyclopédie 1775-1800, Paris, Seuil, 1992, p. 476.

31.C'est le cas de Jean-Baptiste-Louis de ROME DE L'ISLE (1736-1790) qui s'attaque violemment à la cristallographie géométrique de René J. Hauÿ dans sa Cristallographie, ou description des formes propres à tous les corps du règne minéral, dans l'état de combinaison saline, pierreuse ou métallique, avec figures et tableaux synoptiques de tous les cristaux connus, Paris, Imprimerie de Monsieur, 1783, 4 vol.

32.Joseph-Aignan SIGAUD DE LA FOND, Physique générale à l'usage des dames, Paris, 1788, pp. XXVIII-XXIX.

33.James E. MCCLELLAN III, « The Scientific Press in Transition : Rozier's Journal and the Scientific Societies in the 1770 s », Annals of Science, volume 36, $n^{\circ} 5$, septembre 1979, pp. 
425-449. La position de Rozier est reprise par Jean-Claude Delamétherie qui poursuit sous la Révolution la publication du Journal de Physique.

34. La nature considérée sous ses différents aspects ou Journal d'histoire naturelle contenant: tout ce qui a rapport à l'Art vétérinaire, à l'Histoire des différents animaux, au Règne végétal, à la Botanique, au Jardinage et à l'Agriculture, au Règne animal, à l'exploitation des mines, aux usages des différents fossiles et généralement à tous les arts. Ouvrage dans lequel on trouve les Principes élémentaires des sciences, mis à la portée du plus grand nombre des Lecteurs, et les Découvertes qui se font dans les quatre parties du monde, avec des planches gravées en taille douce, par une Société de gens de lettres, rédigé et mis en ordre par l'abbé BERTHOLON, professeur de physique expérimentale des États généraux du Languedoc, des Académies des sciences de Montpellier, Lyon, Bordeaux, Béziers, Dijon, Marseille, Nîmes, Rouen, Toulouse, Valence, Madrid, Rome, Milan, Hesse-Hambourg, Lausanne, Florence, etc. et par M. Boyer, Paris, chez Périsse, 9 tomes (1787-1789). Ce journal se présente comme une « bibliothèque " susceptible de répondre aux attentes de tous ceux qui « n'ont aucune notion des sciences physiques qui ont rapport aux différents aspects sous lesquels on peut considérer la Nature, en acquerront par la lecture de notre Journal, une connaissance suffisante, et que ceux à qui la science n'est pas étrangère, pourront être instruits à l'instant des découvertes, des inventions et des ouvrages nouveaux ». 35. Robert DARNTON, La fin des Lumières. Le mesmérisme et la Révolution, Paris, Perrin, 1984, $220 \mathrm{p}$.

36.Jean et Nicole DHOMBRES, Naissance d'un nouveau pouvoir : sciences et savants en France 1793-1815, Paris, Seuil, 1989, pp. 218 et suiv.

37.Jean-Pierre POIRIER, « Marat et l'Académie des sciences : le différent avec Lavoisier ", in Jean BERNARD et alii, Marat homme de science ?, Paris, Les Empêcheurs de tourner en rond, 1993, pp. 13-34.

38. Ce phénomène a pour conséquence un essor des productions scientifiques qui justifient, en 1791, les propos d'Etienne BARRUEL qui considère qu'il « est temps de simplifier l'étude des sciences et de la débarrasser de cette multitude de livres inutiles si prodigieuse qu'on serait tenté de mettre en question si la découverte de l'imprimé est un bienfait ", Plan d'éducation nationale considéré sous le rapport des livres élémentaires, 1791, pp . 186-187.

39. On comprend ainsi le caractère ambigu de l'hommage que Georges Cuvier adresse à Bernard de Lacépède en 1790 : «J'ai acheté, il y a quelques jours, l'Histoire naturelle des reptiles, de $\mathrm{M}$. de Lacépède, continuateur de Buffon; il a complètement suivi le plan de son prédécesseur dont il a imité le style. Une amélioration importante cependant, c'est qu'il donne une méthode systématique et des caractères tant génériques que spécifiques. Il a adopté les six genres de Linné et en a ajouté deux », Lettres de G. Cuvier à G.M. Pfaff, Paris, Librairie Victor Masson, 1858, « Lettre du 31 décembre 1790 », p. 208. 40.Jonathan MANDELBAUM, « Science and Friendship : The Société Philomathique de Paris, 1788-1815 », art. cit., p. 182.

41. CONDORCET, Eloge de M. Buffon, Paris, Buisson, 1790, p. 42. Plus loin, Condorcet revient sur les fondements de cette science mondaine qu'il dénonce : «M. de Buffon a écrit pour les savants, pour les philosophes et pour le public, et il a su proportionner la clarté de chaque partie au désir qu'il avait d'être entendu d'un nombre plus ou moins grand de lecteurs ", ibid., p. 57.

42.Je mène actuellement une étude sur les procès-verbaux des séances de cette Société entre 1790 et 1798 . 
43. Cit. dans Jonathan MANDELBAUM, « Science and Friendship : The Société Philomathique de Paris, 1788-1815 », art. cit., p. 182.

44.Jean-Luc CHAPPEY, « La Société Nationale des Neuf Sœurs (1790-1793) », Journée d'étude "Sociabilités et réseaux littéraires », Clermont-Ferrand, C.H.E.C., décembre 2003, Paris, Société des Études robespierristes, à paraître.

45.Tribut de la Société nationale des neuf sœurs, Paris, chez Onfroy, 14 juillet 1790, pp. 116-123.

46.Magasin encyclopédique, Paris, de l'imprimerie des Antiquités Nationales, 1er décembre 1792, pp. 6-7.

47.Lia FORMIGARI, Signs, Science and Politics: Philosophies oh Langage in Europe, 1700-1830, Amsterdam, J. Benjamins, 1993, 218 p. ; Jacques GUILHAUMOU, Sieyès et l'ordre de la langue, Paris, Kimé, 2002, 235 p.

48.Alphonse DUPRONT, Qu'est-ce que les Lumières ?, Gallimard, Folio Histoire, 1996, p. 276. 49.Jean-Marc DROUIN, Bernadette BENSAUDE-VINCENT, « Nature for People », in Nicholas JARDINE, James A. SECORD (dir.), Cultures of Natural History, Cambridge University Press, 1996, pp. 408-425.

50.Emma SPARY, «Contrôle du public et vision républicaine dans le Muséum jacobin », in Claude BLANCKAERT et alii [dir.], Le Muséum au premier siècle de son histoire, Éditions du Muséum national d'histoire naturelle, 1997, pp. 457-479 ; idem, Utopia's Garden. French Natural History from Old Regime to Revolution, Chicago, University of Chicago Press, 2000. 51.Yvonne LETOUZEY, Le Jardin des Plantes à la croisée des chemins avec André Thouin (1747-1824), Éd. du Muséum National d'histoire naturelle, 1989, pp. 304-305.

52.Dorinda OUTRAM, « New Spaces in Natural History », in Nicholas JARDINE, James A. SECORD [dir.], Cultures of Natural History, op. cit., pp. 249-265.

53.Selon Roger HAHN, « à l'intérieur de chaque science, la technicité croissante des problèmes liés à chaque discipline et les demandes expérimentales spécifiques à chacune d'entre elles, rendirent la spécialisation plus ou moins inévitables. De plus, le rapprochement des sciences et de ses applications directes tendit à diminuer l'utilité de la science générale face aux demandes spécifiques de la technologie ", L'Anatomie d'une institution scientifique. L'Académie des Sciences de Paris, 1666-1803 [1971], [trad. franç.], Paris, Édition des Archives contemporaines, 1993, p. 392.

54.Patrice BRET, L'État, l'armée, la science. L'invention de la recherche publique en France (1763-1830), Rennes, P.U.R., 2002, 483 p.

55.Henri DESCREMPS, La science sans-culottisée, Paris, chez l'auteur, an II, p. 21. 56.On peut rappeler qu'Henri Descremps s'était illustré dans les années 1780 dans le combat mené contre les « savants-magiciens » qui « se [vantent] ordinairement d'avoir découvert de nouvelles lois dans la nature inconnues jusqu'à lui ; mais il s'en réserve toujours le secret, en assurant que ses connaissances sont du ressort de la physique occulte ", Supplément à la Magie blanche dévoilée, Paris, 1785, pp. 281-282. Voir aussi La magie blanche dévoilée ou explication de tours surprenants qui font depuis peu l'admiration de la capitale et de la province..., Paris, 1784.

57.Henri DESCREMPS, La science sans-culottisée, op. cit., p. 14.

58.Ibid., p. 19.

59.Journal d'instruction publique rédigé par une société de gens de lettres, Paris, Gueffier, 5 thermidor an II-30 frimaire an III, 30 numéros (32 livres par an), Prospectus. 60.Henri DESCREMPS, La science sans-culottisé, op. cit., p. 93 et suiv. 
61.Jean-Luc CHAPPEY, La Société des Observateurs de l'homme. Des anthropologues sous Bonaparte, Paris, Société des Études robespierristes, 2002, 573 p.

62.Daniel RAICHVARG, Jean JACQUES, Savants et ignorants. Une histoire de la vulgarisation des sciences [1991], Point Seuil, 2003, p. 11.

63.Sylvestre-François LACROIX, Sur l'instruction publique. Discours prononcé à la distribution des prix des Écoles centrales du département de la Seine, le 29 thermidor an 8, Paris, de l'imprimerie de Crapelet, an 8, pp. 20-21.

64.Brigitte SCHLIEBEN-LANGE, Franz KNAPSTEIN, « Les Idéologues, avant et après Thermidor ", Annales historiques de la Révolution française, 1988, n²71, pp. 35-57. 65.Gert SCHUBRING, « La réforme du savoir savant : la contribution de Condorcet au premier concours des "livres élémentaires" ", in Pierre CREPEL, Christian GILAIN (dir.), Condorcet. Mathématicien, économiste, philosophe, homme politique, Minerve, 1990, pp. 44-51.

66.Abbé Jacques-Michel coupe (député de l'Oise), Des écoles du peuple ou des premières écoles, thermidor an III (1795), pp. 3-5.

67.Christine BLONDEL, « Haüy et l'électricité : de la démonstration spectacle à la diffusion d'une science newtonienne ", Revue d'histoire des sciences, 1997, 50/3, pp. 265-282.

68.Cf. François AzouVI, L'institution de la raison, Paris, Vrin, 1992, Introduction. 69.Louis DAUBENTON, «Observations sur les noms imposés aux pierres nouvelles découvertes, lues à l'ouverture du Cours de Minéralogie, le 1er floréal an VI », Magasin encyclopédique, an VI, vol. 2, p. 7. Cette critique sera reprise plus tard par P. Denys de Montfort : «Quand on écrit pour la société, on doit lui parler son langage, et ne point affecter d'être étranger au milieu d'elle; on doit désirer se faire entendre de tous et abandonner une nomenclature greco-gothique qui ne peut qu'embrouiller toute chose ", in C.-N.-S. Sonnini de Manoncourt [éd.], Histoire naturelle, générale et particulière, par Leclerc de Buffon, Paris, Dufart, an X (1802), vol. 87, p. 35.

70.Jean-Luc CHAPPEY, « Les sociétés savantes à l'époque consulaire ", Annales historiques de la Révolution française, 1997, n³09, pp. 451-472.

71. Nicolas JOLYCLERC, Principes de la philosophie du botaniste ou Dictionnaire interprète et raisonné des principaux préceptes et des termes que la Botanique, la Physique, la Chimie et l'Agriculture ont consacrés à l'étude et à la connaissance des Plantes, Paris, Ronvaux, an VI, pp. XII-XIII.

72.Ibid.

73.Jean-Luc CHAPPEY, « Le Portique républicain et les enjeux de la mobilisation des arts autour de Brumaire ", in Philippe BOURDIN, Gérard LOUBINOUX (dir.), La Révolution et les arts de la scène, Clermont-Ferrand, 2004, pp. 487-508.

74.Carla HESSE, Publishing and Cultural Politics in Revolutionary Paris, 1789-1810, Oxford, University of California Press, 1991, 296 p.

75.Jean-Luc CHAPPEY, "Catholiques et sciences au début du XIXe siècle », Cahiers d'histoire, 2002, 87, pp. 13-36.

76.Dans ses Mémoires d'outre-tombe, René de CHATEAUBRIAND note que « nos révolutionnaires grands Grecs par nature, ont obligé nos marchands et nos paysans à apprendre les hectares, les hectolitres, les kilomètres, les millimètres, les décagrammes » [Paris, Gallimard, 2000, vol.1, p. 142].

77.Sur ce sujet, voir Jean DHOMBRES, «Culture scientifique et poésie aux alentours de la Révolution française », in Claude BLANCKERT et alii (dir.), Nature, Histoire, Société..., op. cit., 
pp. 341-367 ; on peut également se reporter à l'ouvrage plus ancien de C.-A. Fusil, La poésie scientifique de 1750 à nos jours, Paris, Editions « Scientifica », 1917, 320 p. Les derniers exemples de ce genre sont : Dominique Ricard [Membre associé de l'Institut national], La Sphère, poème en huit chants qui contiennent les Eléments de la sphère céleste et terrestre, ave les principes d'astronomie physique, Paris, Leclère, an V/1796 ; Paul-Philippe Gudin de la Brenellerie [Membre associé de l'Institut national], L'astronomie, poème en trois chants, Auxerre, L. Fournier, an IX (1801).

78.Dorinda OUTRAM, Georges Cuvier : Vocation, Science and Authority in Post-Revolutionary France, Manchester, Manchester University Press, 1984, 299 p. ; Pietro CORSI, Lamarck. Genèse et enjeux du transformisme 1770-1830, C.N.R.S. Éditions, 2001, 434 p. ; Jean-Luc CHAPPEY, La Société des Observateurs de l'homme, op. cit.

79.Marie- Noëlle BOURGUET, « La collecte du monde : voyage et histoire naturelle (fin XVIIIe siècle-début XIXe siècle ", in Claude BLANCKAERT et alii (dir.), Le Muséum au premier siècle de son histoire, op. cit., pp. 163-196.

80.Marie-Noëlle BOURGUET, Déchiffrer la France. La Statistique départementale à l'époque napoléonienne, Paris, Ed. des Archives contemporaines, 1989, 476 p. ; Isabelle LABOULAISLESAGE, Lectures et pratiques de l'espace. L'itinéraire de Coquebert de Montbret, savant et grand commis de l'État (1755-1831), Paris, Honoré Champion, 1999, 753 p.

81.Comme le soulignent Jean et Nicole DHOMBRES, « le phénomène majeur est certainement en cette période de prospérité scientifique que fut l'Empire, le décrochage du public simplement cultivé : il n'était plus question de suivre, même de loin, les problématiques scientifiques en général et on ne croyait plus pouvoir rendre compte de celles-ci en termes usuels ", Naissance d'un nouveau pouvoir..., op. cit., p. 385. 82.Wijnand MINJNHARDT, « Les sciences en Révolution : une affaire d'État ", in Annie JOURDAN, Joep LEERSEN (éd.), Remous révolutionnaires : République batave, armée française, Amsterdam University Press, 1996, p. 218.

83.Bruno BELHOSTE, La formation d'une technocratie. L'École polytechnique et ses élèves de la Révolution au Second Empire, Paris, Belin, 2003, 507 p.

84.Pour Pietro CORDI, « on peut même affirmer qu'entre 1795 et 1805, les partisans de la vieille chimie, de même que les sympathisants de l'histoire naturelle de Buffon [...] espéraient pouvoir reprendre avec succès l'initiative scientifique, et se venger par leurs publications et leur importance culturelle des défaites qu'ils avaient essuyées sur le plan institutionnel », « Buffon sous la Révolution et l'Empire », in Jean GAYON (dir.), Buffon 88, Paris, Vrin, 1992, pp. 639-648.

85.Par exemple Jacques-Louis Nauche rédacteur du Journal du galvanisme, Paris, Buisson, an XI.

86.Jean-Luc CHAPPEY, La Société des Observateurs de l'homme, op. cit.

87.Notons, et ce n'est pas fortuit, que Buisson lance également en germinal an XI, le Journal du galvanisme, de vaccine... voir infra.

88. Bibliothèque physico-économique instructive et amusante par une société de gens de savants, d'artistes et d'agronomes et rédigée par C.S. Sonnini, Paris, chez F. Buisson, an XI (1802), t. I. Ce journal crée en 1788 par Parmentier avait disparu en l'an VI (1798).

89. Comme le souligne Pietro CORSI, « la diffusion des écrits de Buffon, entreprise avec une dévotion sectaire par certains de ses disciples, connut un succès public important et constant, preuve qu'un marché s'offrait à ceux qui, exclus de la science institutionnelle, souhaitaient se tourner vers le grand public des lecteurs d'œuvres 
scientifiques de divulgation ", Lamarck. Genèse et enjeux du transformisme, 1770-1830, op. cit., p. 35.

90.Georges CUVIER, Dictionnaire des sciences naturelles, « Prospectus », Paris, Levrault, vol. I, 1816, pp. VII-VIII.

91.Charles-Nicolas-Sigisbert SONNINI DE MANONCOURT, Nouveau dictionnaire d'histoire naturelle, Paris, Déterville, 1802, vol. 65, p. 37.

92.Ibidem, « Plan de l'ouvrage ».

93. Considérée relativement au public, cette édition ne paraîtra pas moins importante. Ce célèbre médecin est justement mis au nombre des écrivains qui ont appliqué l'éloquence aux sciences physiques, et dont les ouvrages aussi instructifs qu'agréables, ont, comme ceux de Buffon et de Lacépède, un droit égal à l'attention et aux hommages de toutes les classes de lecteurs. [...] Nous terminerons cet avertissement en faisant remarquer que les ouvrages de Vicq-d'Azyr ne doivent pas tout leur prix à un simple mérite littéraire, ainsi que veulent le faire croire quelques personnes intéressées à une semblable opinion; qu'ils sont encore recommandables sous le rapport de la science ; qu'ils se trouvent au niveau des connaissances les plus récentes en physiologie et en médecine, qu'ils contiennent même le germe de plusieurs découvertes ultérieures ; qu'enfin ils offrent des sources d'instruction aussi fécondes qu'agréables [...] », CEuvres de Vicq d'Azyr... par Jacques-Louis Moreau de la Sarthe, Paris, chez L. Duprat-Duverger, an XIII (1805), préface, pp. III et suiv..

94.Tables analytiques et raisonnées des matières et des auteurs pour la nouvelle édition de l'Histoire Naturelle de Buffon rédigée par C.S. Sonnini, t. III, Paris, 1808, t. CXXVII, pp. 343-355 (B.N. S 10 271). Sur les 753 souscripteurs, il faut tenir compte des 339 dont la profession ou le statut social n'est pas indiqué. En outre, comme l'indique Soninni, " plusieurs libraires ont négligé, malgré nos instances réitérées, de nous envoyer la liste de leurs souscripteurs ; de sorte qu'un grand nombre n'y auront pas participé... ». 95. «Bien des gens prétendent que le style n'est d'aucune considération dans les ouvrages d'agriculture. Je ne partage point cette opinion ; je pense, au contraire, qu'offrir les choses utiles sous une forme agréable, c'est engager à les accueillir. D'ailleurs un auteur qui soigne ses écrits fait preuve de déférence pour ses lecteurs, de même qu'un extérieur décent, mais modeste, prouve le respect envers les personnes devant lesquelles on se présente. C'est le pureté et l'élégance du style de l'abbé Rozier, qui ont le plus contribué au prodigieux succès de son Cours complet d'agriculture, livre qui sera recherché dans tous les lieux et dans tous les temps, et que l'on ne parviendra pas plus à faire oublier que l'Histoire naturelle de Buffon ", C.N.S. SONNINI DE MANONCOURT, Traité de l'arachide, Paris, chez D. Colas, 1808, pp. VII-VIII.

96. Comme l'a parfaitement montré Jean-Marc Drouin, la question des applications des sciences naturelles ne se pose pas en terme de transfert d'un savoir spéculatif vers un savoir « utile ». C'est en effet en s'interrogeant sur les applications de la botanique que René-Louiche Desfontaines innove, sur le plan théorique, en proposant une nouvelle méthode de classification des plantes. Voir Jean-Marc DROUIN, « Un savoir utile et attrayant : René Desfontaines (1750-1833) et sa conception de la botanique », Actes du 144 e Congrès national des sociétés savantes, «Scientifiques et sociétés pendant la Révolution et l’Empire », Paris, Éd. du C.T.H.S., 1990, pp. 229-240.

97.Cf. les études consacrées à ces rapports dans Patrice BRET, Marcel DORIGNY (dir.), Sciences et techniques autour de la Révolution française, Annales historiques de la Révolution française, 2000, 4, $230 \mathrm{p}$. 
98.Jean-Pierre CHALINE, Sociabilité et érudition. Les sociétés savantes en France, Paris, Éd. du C.T.H.S., 1995, 270 p.

99.Claude-Isabelle BRELOT, « La noblesse au temps de l'égalité », in Natalie PETITEAU (dir.), Voies nouvelles pour l'histoire du Premier Empire, Paris, La Boutique de l'Histoire, 2003, pp. 215-224.

100.Jean-Baptiste PUjoux, Promenades au Jardin des Plantes, à la ménagerie et dans les galeries du Muséum d'histoire naturelle, Paris, à la Librairie économique, an XII (1803), « Avis de l'auteur».

101.Journal des arts, des sciences et de littérature, Paris, Imprimerie des sciences et des arts, 25 fructidor an $13, \mathrm{n}^{\circ} 439$, p. 300.

102.Claude BLANCKAERT, « La médecine philosophique de F.V. Raspail. Stratégies d'une 'science populaire' ", in Jean POIRIER, Claude LANGLOIS (dir.), Raspail et la vulgarisation scientifique, Paris, Vrin, 1988, pp. 129-198.

103. Cit. in Annie PETIT, « La diffusion des savoirs comme devoir positiviste », Romantisme, $\mathrm{n}^{\circ} 65,1989$-III, p. 9.

104.«L'éditeur à ses souscripteurs », Tables analytiques et raisonnées des matières et des auteurs pour la nouvelle édition de l'Histoire Naturelle de Buffon rédigéé par C.S.Sonnini, Paris, 1808, t. CXXVII, p. 339.

\section{RÉSUMÉS}

La notion de «vulgarisation scientifique » tend à amalgamer des phénomènes qui apparaissent particulièrement divers au risque souvent de la réification. En nous appuyant sur l'étude des transformations théoriques et sociales qui caractérisent le monde des sciences et des savants entre la fin de l'Ancien Régime et l'époque impériale, nous tenterons de préciser les enjeux qui cernent les stratégies de conquête du " public » et de faire apparaître le caractère complexe des relations qui se nouent entre les savants et la société. Cette étude permettra particulièrement de vérifier la singularité du «modèle français » dans le domaine de l'organisation de la science, une singularité qu'il faut rechercher du côté des rapports particuliers que le science entretient avec l'État. À partir de l'analyse des différentes conceptions de la science qui se juxtaposent et s'opposent (science mondaine, utile, sévère et officielle) et de l'étude des étapes qui ponctuent le processus de spécialisation et de professionnalisation des savoirs entre 1780 et 1810, nous montrerons que la "vulgarisation scientifique», telle qu'elle s'impose au XIXe siècle, est le produit de logiques "internes» et «externes» qui rendent nécessaire le croisement entre l'histoire scientifique et l'histoire sociale et politique. Nous verrons ainsi qu'il n'est pas paradoxal d'affirmer que la vulgarisation scientifique n'apparait et n'est possible qu'à condition d'une réelle distanciation entre la science et le public.

Social and Political Aspects of Scientific Vulgarization in the Revolution (1780-1810). The notion of "scientific vulgarization" tends to touch on highly differing issues and often runs the risk of reification. In reviewing the theoretical and social changes affecting the world of science and scientists between the end of the old regime and the Empire, our aim is to identify the issues involved in addressing a "public" and thus highlight the complex links that emerged between the savants and society. This study should enable us to pinpoint the specific nature of the "French 
model" in the way science was organized, a specificness to be found in the special relationship forged by science with the state. By analysing the different conceptions of science that evolved together and often at odds (fashionable science, useful, strict and official science), and scrutinizing the stages that marked the process of specialization and professionalization of knowledge between 1780 and 1810, we endeavour to show that "scientific vulgarization" as it developed in the 19th century was the product of an "internal" and an "external" logic which require a close matching of the history of science and social and political history. It can hence be affirmed without the risk of paradox that scientific vulgarization occurred and could only occur provided a clear margin was preserved between science and the public.

INDEX

Mots-clés : Sciences, vulgarisation, sociétés savantes, classification des savoirs, mondanité

\section{AUTEUR}

JEAN-LUC CHAPPEY

Université de Clermont-Ferrand II / C.H.E.C. - 6, rue Duris 75020 Paris 